\title{
Non-Hermitian Electronics Multipods of Electromagnetically Induced Transparency (EIT) and Absorption (EIA)
}

Senghor TAGOUEGNI ( $\sim$ stagouegni@yahoo.fr)

University of Yaounde I https://orcid.org/0000-0003-0862-5681

Fernande FOTSA-NGAFFO

Universite of Yaounde I

Aurélien KENFACK-JIOTSA

University of Yaounde I

\section{Research Article}

Keywords: non-Hermitian, couplingless, multiple EIT, multiple EIA, ATS.

Posted Date: September 2nd, 2021

DOI: https://doi.org/10.21203/rs.3.rs-772656/v1

License: (c) (i) This work is licensed under a Creative Commons Attribution 4.0 International License.

Read Full License

Version of Record: A version of this preprint was published at Optical and Quantum Electronics on March 1st, 2022. See the published version at https://doi.org/10.1007/s11082-022-03629-4. 


\title{
Non-Hermitian electronics multipods of Electromagnetically Induced Transparency (EIT) and Absorption (EIA).
}

\author{
Senghor Tagouegni ${ }^{1,2, ~}{ }^{*}$, Fernande Fotsa-Ngaffo ${ }^{2,3, \dagger}$, Aurélien Kenfack-Jiotsa ${ }^{2, \S}$ \\ ${ }^{1}$ Laboratory of Mechanics, Materials and Structures, Department of Physics, Faculty of Science, \\ University of Yaoundé I, P.O. Box 812, Yaoundé, Cameroon \\ ${ }^{2}$ Nonlinear Physics and Complex Systems Group, Department of Physics, The Higher Teacher's \\ Training College, University of Yaoundé I, P.O. Box 47, Yaoundé, Cameroon \\ ${ }^{3}$ Institute of Wood technologies, University of Yaoundé I, P.O. Box 306, Mbalmayo, Cameroon. \\ *stagouegni@yahoo.fr \\ †ngafotsa@yahoo.com \\ §kenfack@yahoo.com
}

\begin{abstract}
We study a non-Hermitian electronic dimers system based on an imaginary resistor $(\mathrm{Z})$ in a $(\mathrm{N}+2)$ level atomic multi-pod configuration. Non-Hermitian systems depend on a gain/loss parameter and are specifically marked by a degeneracy exhibited at an exceptional point (EP) separating different phases of complex modes dynamics. Interestingly, the structural characterization and the dispersive properties reveal a broad range of strong coupling where the interplay between the control and the probe field induce a simultaneous EIT, EIA and ATS. Here, by identifying the underlying physical mechanisms, we show that multiple windows of transparency can be strongly enhanced by the incorporation of several dimers in the multipod network. On the other hand, if the pumping field is resonant in the weak regime, multiple EIT and EIA windows result in the number of dimers. Remarkably, the proposed system embedded a multiple coupling mechanism whose modulation induces a couplingless point whereby the energy cross. At this point EIT and related phenomena vanish.
\end{abstract}

Keywords: non-Hermitian $\cdot$ couplingless $\cdot$ multiple EIT $\cdot$ multiple EIA $\cdot$ ATS.

\section{Introduction}

Electromagnetically-induced-transparency (EIT) and related phenomena are prominent examples of coherent interactions between optical fields and multilevel atoms which have spurred the development of new materials with optimized optical properties [1-5]. The coherent preparation produces remarkable changes in the dispersive properties of a medium which leads to quantum interference, namely the fano interferences, between the excitation pathways that control the optical response. The EIT have also been demonstrated to occur via the splitting of energy levels into dressed states by strong coupling fields. In general the transparency of the absorbing medium is increased at large control intensity of the pumping field, leading to the appearance of two dressed states which corresponds to the AutlerTownes splitting (ATS) phenomenon $[6,7]$. Such phenomena have given rise to a 
range of properties including lasing without inversion [8], ultra-slow light [9], stopping of light pulses $[10,11]$ quantum memory [12], enhanced nonlinear optical processes [13], plasmonic sensing with narrow linewidths [14], optical switches [15], just to name but a few. These possibilities opened new avenues for optical information storage and quantum information processing. While EIT renders a narrow spectrum of perfect transmission, its complementary effect known as electromagnetically induced absorption (EIA) defines a band of frequency in which enhanced absorption due to the wave coherence is observed. Traditionally, EIA occurs in a degenerate two-level system [16]. The EIA effect supports some exciting wave propagation concept like the anomalous dispersion, detection of foliage moisture and other types of sensing [17-19].

The realization of EIT in atomic systems has been a difficult task imposed by some restrictions [20]. Interestingly, The EIT effect was demonstrated in classical optical coupled resonators due to its flexible design and easy implementation [21]. However, most of these can be tuned by mechanical alignment making it difficult to realize the dynamic control of the transparency window, which limits the practical application of EIT-like effect. In addition to a three-level type configuration [22], EIT has also been realize in various multilevel configurations [23-26], and quite recently, it was extended to the $\mathrm{N}$ and ( $\mathrm{N}+1)$-level systems [27, 28]. These systems exhibited multiple EIT with interest in the bifurcation of quantum information in multiple channels

Garido Alzar et al. [27] reproduced the EIT behavior experimentally using two linearly coupled LRC circuits. By changing the strength of the coupling parameter, the authors have successfully controlled the response of the system going from the analog of EIT to that of the ATS effect. Joshua Harden [29], Zhengyang Bai and co-authors [30], reproduced double EIT (DEIT) through coupled LRC circuits, in a four-level atomic system, in the Y-inverted and tripod configurations, respectively.

In practice, manipulate LRC circuits in higher frequency regimes may in general lead to miniaturization of devices, higher storage capacities, and larger data transfer rates, but introduces a frequency dependent phase shift and delay effects in two different points of the circuit $[31,32]$ which can hinder the judicious control of EIT. In other hand, from a theoretical point of view, due to the inductor, the dynamics of an LRC oscillator is usually governed by a second order differential equation according to the charge through the capacitor. Hence, solving such equation becomes increasingly complicated when several systems are coupled, so that the slowly varying envelop approximation (SVEA) [33] is often convenient to reduce higher-order equations to the first-order ones, given that EIT is a resonant phenomenon. 
In this paper, we present an alternative approach of designing an electrical analog of multiple Electromagnetically Induced Transparency (EIT) or Absorption (EIA) and related phenomena, based on $(\mathrm{N}+1)$ coupled ZRC circuits in which the inductor $\mathrm{L}$ of the conventional LRC circuit is merely replaced by the imaginary resistor of impedance $\mathrm{Z}$, whose the main characteristic is the ability to generate frequency independent phase shift, unlike inductor/capacitor reactive elements, which can only do this task when they are associated with a non-zero frequency $[31,32]$. The ways of realizing the imaginary resistor have been suggested in the literature using a gyrator [34-37]. The imaginary resistor impedance's is frequency independent $\left(Z=j r\right.$, with $\left.j^{2}=-1\right)$, allowing to properly manipulate the lower and higher frequencies as desired, therefore leading to a system of first order ordinary differential equations, which facilitates the analysis of $\mathrm{N}$ coupled circuits. Besides, the ZRC oscillator has a natural frequency $\omega_{0}=1 / r C$, which can be positive or negative according to the real value of the resistance $r$ associated to the imaginary resistor and the capacitance $C$ of the circuit. This allows to reproduce with a so called ZRC multi dimer, for the first time to our knowledge, the analog of quantum interference phenomena with negative frequencies of the circuit. By manipulating couplings in presence, tuning the gain/loss parameter linking parts of our circuit, offered us the possibility to merge from single to multiple EIT, EIA and ATS windows.

Our paper is organized as follow: in section 2, we present the model equations and the eigenmodes analysis. Here, a structural characterization allows us on the one hand to identify for the system a weak and a strong coupling regimes, and on the other hand, to emphasise the couplingless point (CPLP). In section 3, the steady state dynamic and dispersive properties of the systems are investigated. After that we arrive at the section 4 which summarizes the paper results.

\section{Equations of model and eigenmodes analysis}

We consider a ZRC multi dimer obtained by coupling $(\mathrm{N}+1)$ oscillators ZRC as shown in Fig. 1 (a). Each $Z_{i} R_{i} C_{i}$ circuit consists of a linear capacitor $C_{i}$, a real resistor $R_{i}$ and an imaginary resistor of impedance $Z_{i}$ where $Z_{i}=j r_{i}\left(j^{2}=-1\right)$, all arranged in series. The sub-index $i=(0 \ldots N)$ indicates the oscillator's number, and $N,\left(\begin{array}{ll}N^{3} & 1\end{array}\right)$ is the total number of cells coupled to the main loop oscillator $Z_{0} R_{0} C_{0}$ for which a harmonic voltage source $u(t)$ has been added in series with the resistor $R_{0}$. The coupling between loops is realized thanks to the capacitor $C_{C}$ and another imaginary resistor $Z_{C}\left(Z_{C}=j r_{C}\right)$, also arranged in series and belonging to the set of circuit meshes. 
(a)

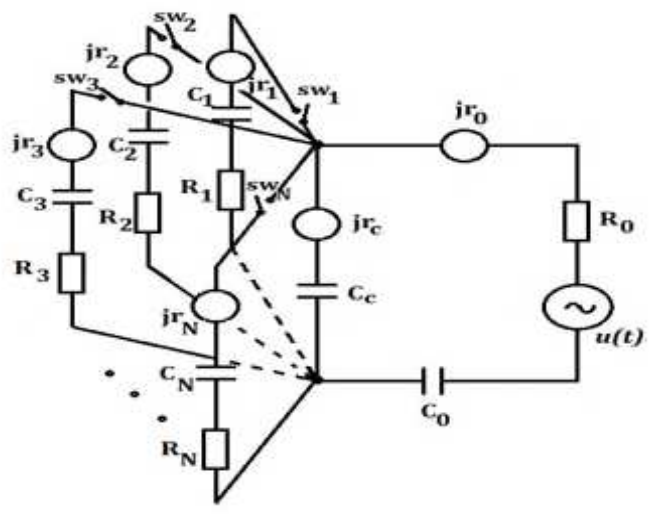

(b)

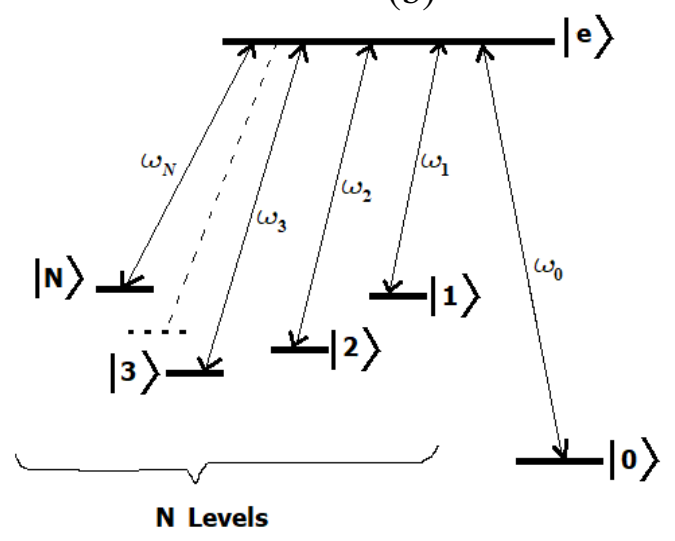

Fig. 1: (a) ZRC Multi dimer circuit used to mimic a single and multiple EIT, ATS and EIA windows. The circuit is based on the imaginary resistor. (b) Energy diagram of a $(\mathrm{N}+2)$ level atomic system in a multi-pod type configuration consisting of $(\mathrm{N}+1)$ lower levels $|i\rangle(i=0 . . N)$ of which $\mathrm{N}$ pumping lasers couple near resonantly each state to the upper state $|\mathrm{e}\rangle$, while a probe laser couples level $|0\rangle$ to $|\mathrm{e}\rangle$.

The model in addition to group a considerable number of cells and couplings, presents some particularities compared to the analogous models based on LRC circuits that were proposed in the literature [28-30]. Indeed, the inductor has been replaced by an imaginary resistor, the main characteristic of which is to generate an impedance and a phase shift independently of the frequency, which is not the case for the usual reactive elements. In particular, the so called ZRC multi dimer leads to a system of coupled first order ordinary differential equations, which are easily manipulated. Moreover, the system can support negative frequencies, without alterating it operating mode, therefore it opens avenue to investigate various quantum phenomena through positive and negative frequencies. The imaginary resistor can be realized directly using an ideal transformer [34].

Fig. 1(b) illustrates a $(\mathrm{N}+2)$ level atomic system in a multi-pod type configuration, where the $(\mathrm{N}+1)$ lower states $|i\rangle$ which could be degenerated or not, are coupled to a single excited upper level state $|e\rangle$. Imagine that the atomic system is initially prepared to be in the particular lower level $|0\rangle$ where it interacts with a probe laser which serves to couple this level to the upper level $|\mathrm{e}\rangle$. Adding $\mathrm{N}$ sources light of frequency $\omega_{i}$ driving each atomic transition from $|i\rangle$ to $|\mathrm{e}\rangle$, the absorption spectrum of the probe laser is modified once the $\mathrm{N}$ control fields are simultaneously interacting with the medium. It results interferences between different transitions pathways of the atom. When $\mathrm{N}=1$, this interference phenomenon is known as electromagnetically induced transparency (EIT), and it occurs one dip in the absorption spectrum. It corresponds to double EIT (DEIT) when $\mathrm{N}=2$, accordingly, two dips are simultaneously observed. Hence, if $\mathrm{N}$ is greater, the phenomenon is called multiple EIT and consequently $\mathrm{N}$ dips appear in the absorption spectrum of the probe laser. 
A correspondence can be made between the ZRC multi dimer and the atomic system so described. In this analogy, the loop $Z_{0} R_{0} C_{0}$ of frequency $\omega_{0}$ models the atom and the harmonic voltage $u(t)$ which drives it directly, replaces the probe laser field. Once the switch $S W i$ is closed, each loop $Z_{i} R_{i} C_{i}(i \neq 0)$ of frequency $\omega_{i}$ acting on the main loop simultaneously as the voltage, then models a pumping (control) laser field.

The switches $\mathrm{SW} i, i=(0 \ldots N)$ being closed, applying the Kirchhoff's laws to the circuit allows to obtain the following coupled equations:

$$
\left\{\begin{array}{l}
\left(\beta_{0}+j\right) \frac{d Q_{0}}{d t}-j \beta_{c 0} \sum_{n=1}^{N} \frac{d Q_{n}}{d t}+\omega_{0} Q_{0}-\Omega_{0} \sum_{n=1}^{N} Q_{n}=u / r_{e o}, \quad \text { for } i=0 \\
\left(\beta_{i}+j\right) \frac{d Q_{i}}{d t}-j \beta_{c i}\left(\frac{d Q_{0}}{d t}-\sum_{\substack{n=1 \\
n \neq i}}^{N} \frac{d Q_{n}}{d t}\right)+\omega_{i} Q_{i}-\Omega_{i}\left(Q_{0}-\sum_{\substack{n=1, n \neq i}}^{N} Q_{n}\right)=0, \text { for } i \neq 0
\end{array}\right.
$$

where $j=\sqrt{-1}, i=0 . . N, \beta_{i}=R_{i} / r_{e i}, \beta_{c_{i}}=r_{c} / r_{e i}, \omega_{i}=1 / r_{e i} C_{e i}, \Omega_{i}=1 / r_{e i} C_{c}, r_{e i}=r_{i}+r_{c}$ and $C_{e i}=C_{i} C_{c} /\left(C_{i}+C_{c}\right)$.

$Q_{i}$ and $d Q_{i} / d t$ represent the charges and the current through each loop, respectively. The equivalent loops resistance $r_{e i}$ and capacitance $C_{e i}$ are the series combination of the real values of resistances associated to the imaginary resistors $\left(Z_{i}\right.$ and $\left.Z_{C}\right)$ and the capacitors $\left(C_{i}\right.$ and $\left.C_{c}\right)$, respectively. $\beta_{i}=R_{i} / r_{e i}$ is the non-Hermitian parameter (again called gain/loss parameter) responsible to the amplification or the damping into the loops of natural frequencies $\omega_{i}=1 / r_{e i} C_{e i}$. Remarkably, when $r_{e i}$ and $R_{i}$ have the same signs (i.e. $\beta_{i}>0$ ), the loop is said "loss" loop, otherwise, it is called the "gain" loop. The way to realize the negative resistor can be found in ref. [38]. Table 1 gives us the possible signs of the circuitry elements of a $Z_{i} R_{i} C_{i}$ loop. In the following, we consider that the gain/loss values into the coupled loops $\left(\beta_{i}, i \neq 0\right)$ are linking to the gain/loss of the main loop $\left(\beta_{0}\right)$ as:

$$
\varepsilon=\frac{\beta_{i}}{\beta_{0}}
$$

\begin{tabular}{|l|l|l|l|l|l|l|l|l|}
\hline \multirow{2}{*}{} & \multicolumn{2}{|c|}{$r_{C}$} & \multicolumn{2}{c|}{$C_{C}$} & \multicolumn{2}{c|}{$C_{e i}$} & \multicolumn{2}{c|}{$R_{i}$} \\
\cline { 2 - 8 } & $r_{C}>0$ & $r_{C}<0$ & $C_{C}>0$ & $C_{C}<0$ & $C_{e i}>0$ & $C_{e i}<0$ & $R_{i}>0$ & $R_{i}<0$ \\
\hline$r_{e i}>0$ & $\beta_{c i}>0$ & $\beta_{c i}<0$ & $\Omega_{i}>0$ & $\Omega_{i}<0$ & $\omega_{i}>0$ & $\omega_{i}<0$ & $\beta_{i}>0$ & $\beta_{i}<0$ \\
\hline$r_{e i}<0$ & $\beta_{c i}<0$ & $\beta_{c i}>0$ & $\Omega_{i}<0$ & $\Omega_{i}>0$ & $\omega_{i}<0$ & $\omega_{i}>0$ & $\beta_{i}<0$ & $\beta_{i}>0$ \\
\hline
\end{tabular}

Table 1. Possible signs of the circuitry elements of a $Z_{i} R_{i} C_{i}$ loop.

In this section, the circuit is investigated in absence of the voltage source

\subsection{ZRC dimer}


The ZRC dimer is obtained by coupling only two oscillators $Z_{i} R_{i} C_{i}(i=0,1)$ as schematically described by the Fig. 1(a) when $\mathrm{N}=1$. We assume that both loops have the same natural frequency $\omega_{0}=1 / r C$, where $r=r_{e i}$ and $C=C_{e i}, \omega_{0}$ being positive or negative (see Table 1 for the conditions). As in ref. [39], the active coupling can be used to refer to the ZRC dimer. For convenience, for the dimer Z, only the imaginary resistive coupling $\left(Z_{C}\right)$ is active while in the case of dimer $\mathrm{ZC}$, the series combination $Z_{C}-C_{C}$ is active. Rewriting Eq. (1) for $i=0,1$ and assuming the harmonic solutions $Q_{i}(t)=A_{i} e^{j \omega t}+$ c.c for the charges leads to the following matrix representation:

$$
\left(\begin{array}{cc}
\delta+j \omega \beta_{0} & \omega \beta_{c}-\Omega \\
\omega \beta_{c}-\Omega & \delta+j \omega \beta_{1}
\end{array}\right)\left(\begin{array}{l}
A_{0} \\
A_{1}
\end{array}\right)=\left(\begin{array}{l}
0 \\
0
\end{array}\right),
$$

where $\beta_{C}=r_{C} / r, \Omega=1 / r C_{C}=\kappa \omega_{0}, r=r_{0,1}+r_{c}, \omega_{0}=1 / r C$ and $\delta=\omega_{0}-\omega$ is the frequency detuning.

We are interested of the behavior of the system near the resonance. For that, we use the approximation $\left|\omega-\omega_{0}\right|=\omega, \beta \omega \approx \beta \omega_{0}$ [40]. Therefore, Eq. (3) can be rewritten as:

$$
\left[\omega_{0}\left(\begin{array}{cc}
1+j \beta_{0} & c \\
c & 1+j \beta_{1}
\end{array}\right)-\omega\left(\begin{array}{ll}
1 & 0 \\
0 & 1
\end{array}\right)\right]\left(\begin{array}{l}
A_{0} \\
A_{1}
\end{array}\right)=\left(\begin{array}{l}
0 \\
0
\end{array}\right),
$$

where $c=\beta_{c}-\kappa$ is the effective coupling of the system. Eq. (4) has non-trivial solutions if the determinant of the left-most term vanishes. Then, we need to solve the characteristic equation $|H-\omega I|=0$, where $I$ is the identity matrix and $H$ is a nonHermitian Hamiltonian [40] written as:

$$
H=\omega_{0}\left(\begin{array}{cc}
1+j \beta_{0} & c \\
c & 1+j \beta_{1}
\end{array}\right) .
$$

Through diagonalization, the eigenfrequencies $\omega_{ \pm}$of the system dimer can be obtained as:

$$
\omega_{ \pm}=\omega_{0}\left(1+j \mathrm{~B} \pm \sqrt{c^{2}-\beta^{2}}\right),
$$

with $\mathrm{B}=\left(\beta_{1}+\beta_{0}\right) / 2=\beta_{0}(\varepsilon+1) / 2, \beta=\left(\beta_{1}-\beta_{0}\right) / 2=\beta_{0}(\varepsilon-1) / 2, c=\beta_{c}-\kappa$ and $\kappa=\Omega / \omega_{0}$. The eigenfrequencies obtained in Eq. (5) are analyzed considering two cases: the case $\omega_{0}>0$ and the case $\omega_{0}<0$. Since one innovation of this paper is the use of the negative frequencies, in the following analysis, the natural frequency is set negative $\left(\omega_{0}<0\right)$, unless otherwise specified.

In fig. 2, the real (first column) and the imaginary (second column) parts of the normalized eigenfrequencies $\omega_{ \pm} /\left|\omega_{0}\right|$ of the ZRC dimer are represented in 3D top view as a function of the active coupling parameter and the gain/loss ratio $\varepsilon$, in the case of dimer Z where $c=\beta_{c}$ (plots (a), (b)) and in the case of dimer ZC with $\kappa=0.75$ (plots (d), (e)). In the third column, the cross section of both the real (solid) and imaginary (dashed) parts are also made for different values of $\varepsilon$ (plot (c) for the dimer $\mathrm{Z}$ and plot (f) for the dimer ZC). The values of $\varepsilon$ and the corresponding 
colors are indicated on the plots. In addition, for all plots we have set $\beta_{0}=0.5$ and $\omega_{0}$ is negative. As we can see on figures, the eigenfrequencies $\omega_{ \pm}$are mostly complex and display a transition at two particular points known as the exceptional points (EPs), where the effective coupling strength $c=c_{t h}$ obeys to $\left|c_{t h}\right|=|\beta|$. This effective coupling threshold is shown in the 3D plots ((a), (b) (c) and (d)) with the dashed purple color and it can be remarked that $c_{t h}$ cancels when $\varepsilon=1$ in the case of dimer Z (only the imaginary coupling is active). Remarkably, in the case of dimer $\mathrm{ZC}$, the coupling threshold does not vanishes, but reaches the critical value when $\varepsilon=1$. This allows us to distinguish two coupling regimes for the system: the regime between the EPs $(|c|<|\beta|)$ called weak-driving regime, characterized by the coalescence of the real parts of the eigenfrequencies and the splitting of their imaginary parts, and the regime of coupling located outside the EPs, namely the strong coupling regime in which $|c|>|\beta|$. Here, the imaginary parts of the eigenfrequencies do cross while their real parts are split. Also, remark from plots that the EPs are symmetric from zero for the dimer $\mathrm{Z}$, which is not the case for the dimer ZC, where the symmetry axis is shifted according to the critical value $\beta_{C}=\kappa$ which behaves as an equilibrium point. For the parity time symmetric (PTS) case ( $\varepsilon=-1$ ) where the gain compensates the losses, we notice a totally conservation of the energy in the strong regime where the eigenfrequencies are real.
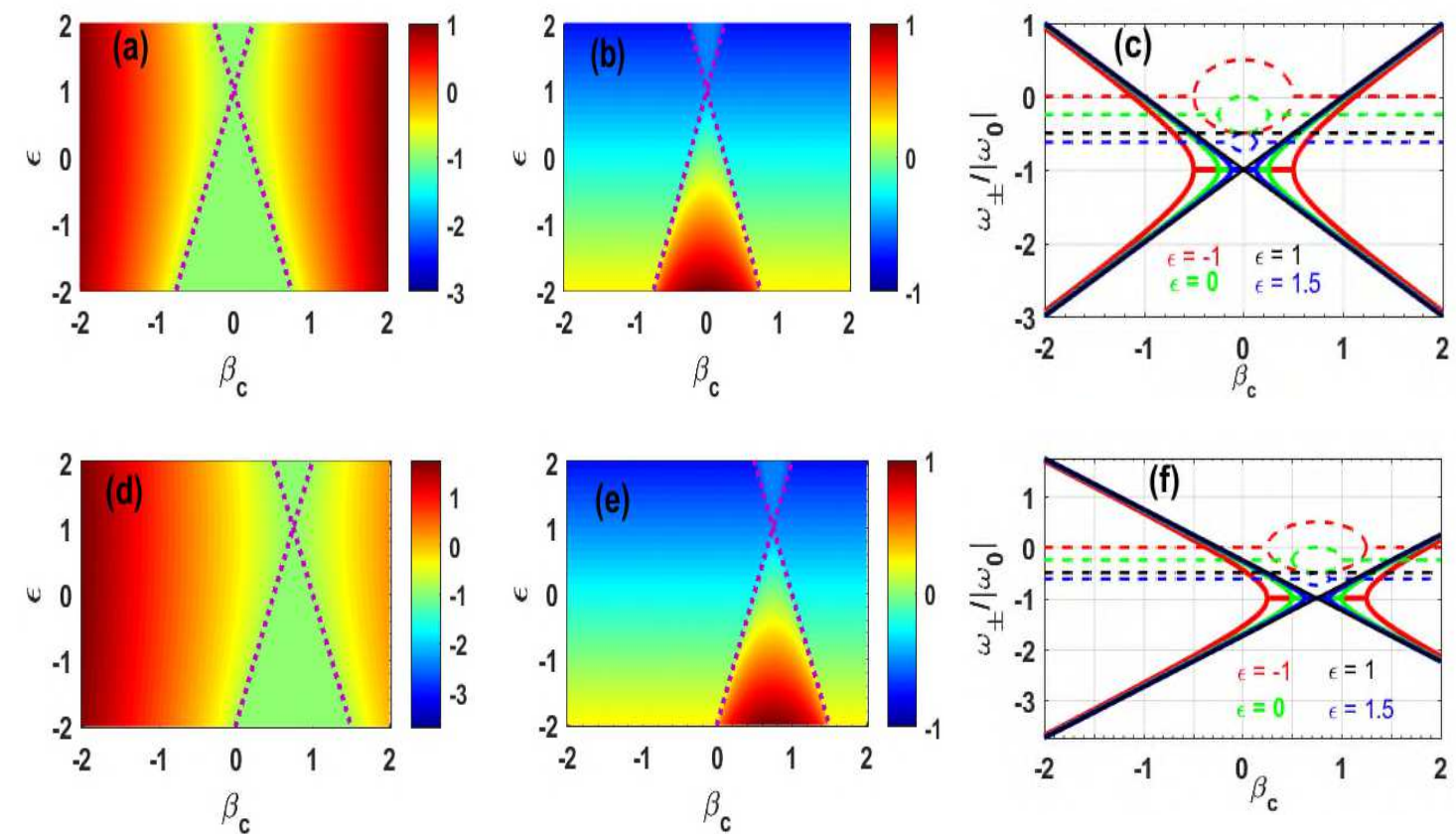

Fig. 2 : 3D top view of the real (first column) and imaginary (second column) parts of the normalized eigenfrequencies $\omega_{ \pm} /\left|\omega_{0}\right|$ of the ZRC dimer as function of the active coupling parameter and the gain/loss ratio $\varepsilon$, in the case of dimer $\mathrm{Z}$ of which $c=\beta_{c}$ (plots (a), (b)) and the case of dimer ZC with $\kappa=0.75$ (plots (d), (e)). The coupling threshold $c_{t h}$ is shown here with the dashed purple color. Third column: cross section of the real (solid) and imaginary (dashed) parts of $\omega_{ \pm}$made for different values of $\varepsilon$ : (c): dimer $\mathrm{Z}$ and (f): dimer ZC with $\kappa=0.75$. The values of $\varepsilon$ and the corresponding colors are indicated on the plots. For all plots we set $\beta_{0}=0.5$. 


\section{-The Couplingless point (CPLP)}

The couplingless point (CPLP) is a particular point where a combination of coupling parameters of the dimer ZC allows a cancellation of the effective coupling $c$. It is easily to find the CPLP when the gain/loss ratio is equal to one $(\varepsilon=1)$. The couplingless condition is given in Eq. (6).

$$
c=0 \Leftrightarrow \kappa=\beta_{c}
$$

To well characterize the CPLP and the coupling regimes evoked, let us assume that the coupled loops oscillate with different natural frequencies. For convenience, we consider that the frequencies are $\omega_{0}$ and $\omega_{0}(1+\Delta)$ where $\Delta$ is a small variation. In this case, the eigenfrequencies found in Eq. (5) can be rewritten as:

$$
\omega_{ \pm}=\omega_{0}\left(1+\frac{\Delta}{2}+j \mathrm{~B} \pm \frac{1}{2} \sqrt{\Delta^{2}-4 \beta^{2}+4 c^{2}+j 4 \Delta \beta}\right)
$$

Fig. 3 shows the real parts of the eigenfrequencies $\omega_{ \pm}$(green color for $\omega_{+}$and red color for $\omega_{-}$) obtained in this context (see Eq. (7)) as function of the variation $\Delta$ in the case of dimer Z and dimer ZC. Considering the values of $\varepsilon=0$ and $\kappa=0.75$ constants, we can vary the parameter $\beta_{C}$ such as to switch from the weak to the strong coupling. The quantity $\operatorname{Re}\left(\omega_{+}-\omega_{-}\right)$representing the frequency splitting is indicated by a dashed black color.

In absence of any coupling i.e. $\beta_{C}=\kappa=0(c=0)$, Fig. 3 (a) shows that, as the value of $\Delta$ is increasing, the frequency of the main loop remains constant equal to $\omega_{0}$, while the frequency $\omega_{1}$ decreases. Both curves intersect at $\Delta=0$ where the frequency splitting is zero. However, when the weak coupling is established between both oscillators of the dimer Z (Fig. 3(b)) or the dimer ZC (Fig. 3(d)), one of the frequencies decreases while another remains constant as $\Delta$ increases. We remark that both frequencies intersect at $\Delta=0$ where the splitting is equal to zero; but after that, the frequencies interchange their role. In fact, the frequency which was constant before the value $\Delta=0$ now decreases as $\Delta$ is increasing, whereas the other remains constant equal to $\omega_{0}$. On the contrary, when a strong coupling is made between oscillators, whatever the dimer Z (Fig.3(c)) or the dimer ZC (Fig.3(e)), we remark that both eigenfrequencies decrease as the parameter $\Delta$ is increasing. However, they show the anticrossing which is the main characteristic of the strong coupling at $\Delta=0$ where the frequencies are split. As defined above, the CPLP is a point belonging to the weak coupling domain where the effective coupling vanishes i.e. $\beta_{C}=\kappa(c=0)$. However, there, it is remarked (see Fig. 3(f)), that one eigenfrequency remains equal to $\omega_{0}$ while the other one decreases as $\Delta$ increases from -2 to zero, where they intersect exactly at $\Delta=0$, as in the case illustrated in Fig. 3(a). The inset plot shows the imaginary parts of the eigenfrequencies in that point, which allows to distinguish it to the case obtained in (a) in absence of any coupling. Remarkably, it is important to recall that the CPLP only exits in the dimer ZC including two types of couplings. 


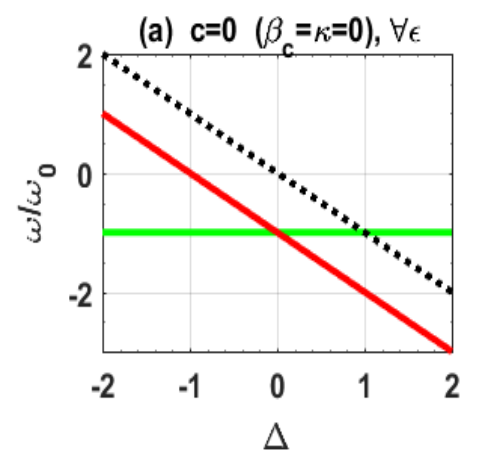

(d) weak coupling

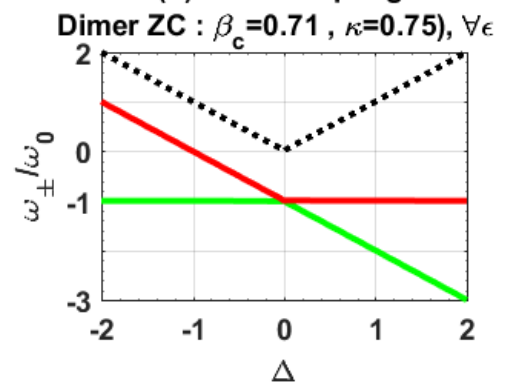

(b) weak coupling

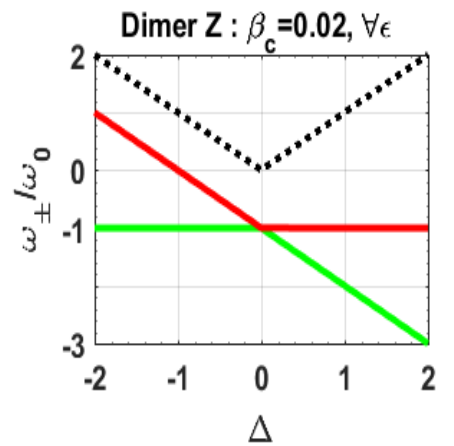

(e) strong coupling

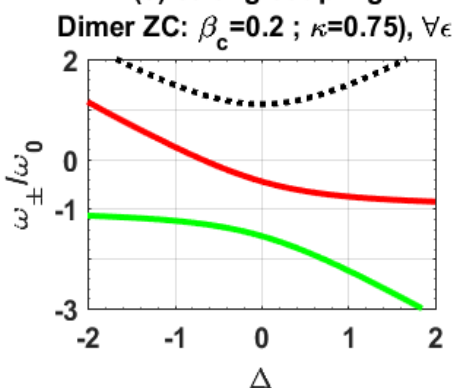

(c) strong coupling

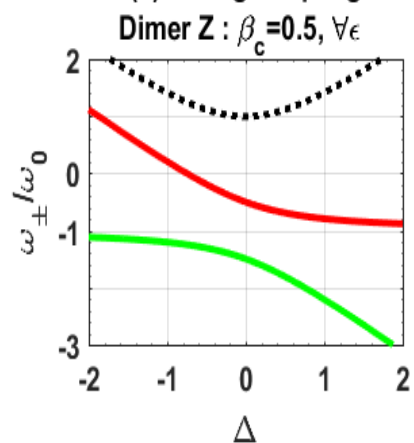

(f) CPLP

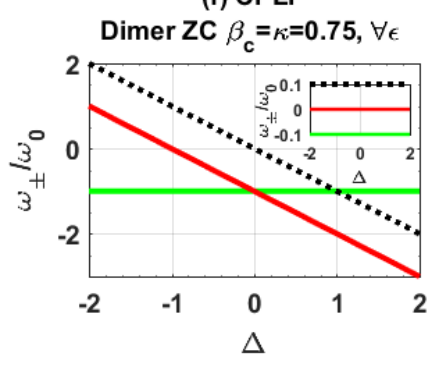

Fig.3: Eigenfrequencies of dimer as function of the variation $\Delta$. (a) Uncoupled loops $\left(\beta_{C}=\kappa=0(c=0)\right.$ ). Dimer Z in the regime of weak (b) and strong (c) coupling. Dimer ZC in weak (d) and strong (e) coupling regime. (f) Dimer ZC at the couplingless point $\left(\beta_{C}=\kappa=0.75(c=0)\right.$ ). In all plots, $\beta_{0}=0.1, \omega_{0}<0$ and $\varepsilon=0$.

\subsection{ZRC multi dimer}

Let us now focus on the ZRC multi dimer scheme, as depicted in Fig. 1(a) when $N>1$. We consider now that each loop oscillates with its own natural frequency $\omega_{i}(i=0 . . N)$, which differs from one loop to another. As described above for the ZRC dimer, in the following, the active coupling in place and the number $\mathrm{N}$ of loops coupled to the main loop, are used to refer to the ZRC multi dimer. In this way for example, the bi dimer $\mathrm{C}$ is the circuit of which the active coupling is capacitive $\left(C_{C}\right)$ and where $\mathrm{N}=2$, the tri dimer $\mathrm{Z}$ is the one of imaginary resistive coupling $\left(\beta_{C}\right)$ with $\mathrm{N}=3$, whereas the tetra dimer $\mathrm{ZC}$ is the circuit of which four loops are coupled to the main loop via both the capacitor and the imaginary resistor. Looking for the harmonic solutions $Q_{i}(t)$, Eq. (1) describing the motion of the ZRC multi dimer can be rewritten in the following matrix representation, in absence of the harmonic voltage:

$$
\left(\begin{array}{ccccc}
\chi_{0} & c_{0} & c_{0} & \mathrm{~L} & c_{0} \\
c_{1} & \chi_{1} & -c_{1} & \mathrm{~L} & -c_{1} \\
c_{2} & -c_{2} & \chi_{2} & \mathrm{~L} & -c_{2} \\
\mathrm{M} & \mathrm{M} & \mathrm{M} & & \mathrm{M} \\
c_{N} & -c_{N} & -c_{N} & \mathrm{~L} & \chi_{N}
\end{array}\right)\left(\begin{array}{c}
A_{0} \\
A_{1} \\
A_{2} \\
\mathrm{M} \\
A_{N}
\end{array}\right)=\left(\begin{array}{l}
0 \\
0 \\
0 \\
\mathrm{M} \\
0
\end{array}\right),
$$


where $(i=0 . . N), c_{i}=\beta_{c_{i}}-\kappa_{i}, b_{C_{i}}=r_{C} / r_{e i}, \kappa_{i}=\Omega_{i} / \omega_{0}, \chi_{i}=\delta_{i} / \omega_{0}+j \beta_{i}, \delta_{i}=\omega_{i}-\omega, \beta_{i}=R_{i} / r_{e i}$, $\omega_{i}=1 / r_{e i} C_{e i}, r_{e i}=r_{i}+r_{c}$ and $C_{e i}=C_{i} C_{C} /\left(C_{i}+C_{C}\right)$.

We want to find the eigenfrequencies of the multi dimer. To simplify the study, we assume that the imaginary resistances are constant i.e. $r_{i(i=0 . . N)}=r$, only capacitances are distinct. Based on this, $\beta_{C_{i}}=\beta_{C}, \kappa_{i}=\kappa$ and it results that $c_{i}=c=\beta_{C}-\kappa$. Under the approximation $|\omega-\varpi|=\omega, \beta_{i} \omega \approx \beta_{i} \varpi=\beta_{i} \omega_{0}$ where $\varpi=\omega_{0}$ is the mean frequency, and using a few steps of algebra, the determinant of the left most term of Eq. (8) can be obtained as:

$$
\begin{aligned}
& D(\omega)=\prod_{i=0}^{N} \chi_{i}-N c^{N+1}-\sum_{p=1}^{N} \sum_{i=0}^{N-p+1} \sum_{k=i+1}^{N-p+2} \mathrm{~L} \sum_{s=i+q}^{N-p+q}(N-p) c^{N-p+1} \chi_{i} \chi_{k} \mathrm{~L} \chi_{s}, \\
& 1 \leq p \leq N, 2 \leq n \leq N, \mathrm{~N} \geq 1, \quad q \geq 2, u \geq 3
\end{aligned}
$$

The eigenfrequencies of the systems are the roots of $D(\omega)$. As the number $\mathrm{N}$ of the coupled loops increases from $\mathrm{N}=2$ to $N>2$, generally it becomes more difficult to find analytically these roots, given that the expression of $D(\omega)$ combines complex terms. For this reason, we have numerically calculated the roots of which the real and the imaginary parts are illustrated in Fig. 4 as function of the imaginary resistive coupling parameter $\beta_{C}$ in the case of the multi dimer ZC (with $\kappa=0.75$ ), assuming the main loop frequency $\omega_{0}$ negative and the gain/loss in the parts of the circuits such that $\beta_{i(i=1 . . N)}=\varepsilon \beta_{0}$ (where $\beta_{0}=0.5$ (loss loop) and $\varepsilon=0$ ). In addition, we have considered the natural frequencies of the loops such as: for the bi dimer ZC $(\mathrm{N}=2)$ : $\omega_{1}=\omega_{0}-0.1 \omega_{0}$ and $\omega_{2}=\omega_{0}+0.1 \omega_{0}$; tri dimer ZC $(\mathrm{N}=3): \omega_{1}=\omega_{0}-0.1 \omega_{0}, \omega_{2}=\omega_{0}+0.02 \omega_{0}$ and $\omega_{3}=\omega_{0}+0.1 \omega_{0} ;$ tetra dimer ZC $\quad(\mathrm{N}=4): \omega_{1}=\omega_{0}-0.1 \omega_{0}, \omega_{2}=\omega_{0}+0.1 \omega_{0}$ $\omega_{3}=\omega_{0}-0.2 \omega_{0}$ and $\omega_{4}=\omega_{0}+0.2 \omega_{0}$, where $\omega_{0}$ is the natural frequency of the main loop. As one can rapidly perceive, the figure shows a close dependence between the number of coupled cells $\mathrm{N}$ and the roots of $D(\omega)$. In the real parts of the roots demonstrated in Figs. 4(a), 4(b) and 4(c), for $\mathrm{N}=2,3$ and 4, respectively, it is seen that around the CPLP for which $\beta_{C}=\kappa=0.75$, the roots keep the same values as those of uncoupled oscillators and then display the avoided crossing. This is confirmed by the inset in Fig. 4(a) obtained with the bi dimer Z, where the same behavior occurs around a zero imaginary resistive coupling. As the absolute value of the coupling $\left|\beta_{C}\right|$, increases, one notice that one of the real part of the roots separates from the others, while they get closer; accordingly, a large splitting is observed between this mode and the others as the coupling is further increases. On the contrary, the imaginary parts of the roots depicted on Figs. 4(d), 4(e) and 4(f) for the cases $\mathrm{N}=2,3$ and 4, respectively, show a behavior little different to what is exhibited by the real parts. Indeed, around the CPLP, the imaginary parts of the roots are closed to zero, except one of which the value is high. Far of the CPLP, the 
imaginary parts of the modes evolve asymptotically each towards a constant value, which can be zero.
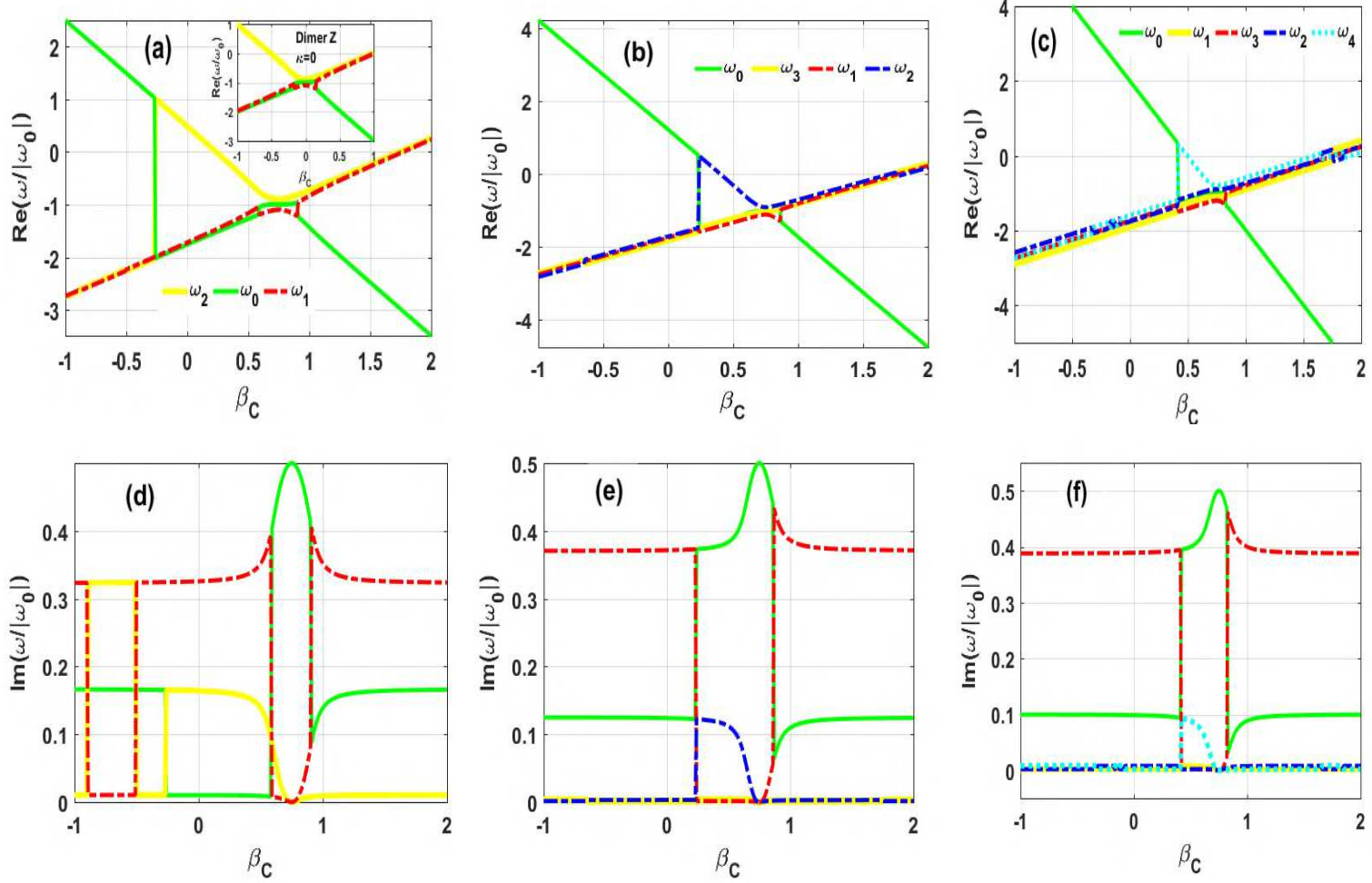

Fig.4: The normalized roots of $D(\omega)$ as function of the imaginary resistive coupling parameter $\beta_{C}$ for the multi dimer $\mathrm{ZC}$ (with $\kappa=0.75$ ). First column: bi dimer $\mathrm{ZC}(\mathrm{N}=2)$. Second column: tri dimer $\mathrm{ZC}(\mathrm{N}=3)$ and in last column: tetra dimer $\mathrm{ZC}(\mathrm{N}=4)$. The upper row shows the real parts of the roots $\left(\operatorname{Re}\left(\omega /\left|\omega_{0}\right|\right)\right)$ and the down row shows their imaginary parts $\left(\operatorname{Im}\left(\omega /\left|\omega_{0}\right|\right)\right)$. The other plots parameters are: $\varepsilon=0, \beta_{0}=0.5$ and $\omega_{0}<0$. The inset plot in (a) corresponds to the case of bi dimer $Z(\kappa=0)$. The inset shows that the frequencies of the bi dimer ZC at the CPLP $\left(\beta_{C}=\kappa\right)$ behaves as those of the dimer $\mathrm{Z}$ around the zero coupling.

\section{Steady state dynamic and dispersive properties}

In this section, we are interested to the dynamic of the system in presence of the harmonic voltage source $u(t)$.

\subsection{Single ZRC oscillator}

Let's get interest first to the main oscillator loop $Z_{0} R_{0} C_{0}$, in absence of any coupling (SW $i$ off). The first row of Eq. (1) is rewritten as:

$$
\left(\beta_{0}+j\right) \frac{d Q_{0}}{d t}+\omega_{0} Q_{0}=u / r_{e 0},
$$

where $u(t)=V_{0} e^{j \omega t}+$ c.c with c.c the complex conjugate, $\omega$ is the harmonic voltage frequency, $V_{0}$ its amplitude and $\beta_{0}^{-1}=\left(R / r_{e 0}\right)^{-1}$ is the quality factor. It has been shown in ref. [32] that the quality factor displays an important role to determine the nature of the oscillations of a ZRC oscillator. For example, when the frequency $\omega_{0}$ is 
positive $\left(\omega_{0}>0\right)$, the oscillations are damped during the time for the loss loop of which $\beta_{0}{ }^{-1}>0$, but are amplified for the gain loop where $\beta_{0}{ }^{-1}$ is negative. On the contrary, when the frequency of the loop is negative $\left(\omega_{0}<0\right)$, the oscillations are amplified in the loss loop while they are damped into the gain loop. However, if the quality factor is large $\left(\beta_{0}^{-1} \rightarrow \infty\right)$, the amplitude of oscillations remains constant whatever the sign of the frequency, since the oscillator ZRC seems as the simple ZC oscillator.

The general solution of Eq. (10) can be written in the form:

$$
Q_{0}(t)=a e^{-w_{0} t /\left(b_{0}+j\right)}+\left(A e^{j w t}+\text { c.c }\right),
$$

where, $A=V_{0} / \hat{e}_{e 0}\left(d+j b_{0} w\right)$ ì is a complex amplitude, c.c the complex conjugate, $\delta=\omega_{0}-\omega$ is the frequency detuning and $\alpha$ is a constant amplitude. In the steady state regime, the first term of $Q_{0}(t)$ can be neglected and we can only consider that $Q_{0}(t) » A e^{j w t}+$ c.c. Hence, we can evaluate the dissipated power through the loop as $P_{0}(t)=\left(V_{0} e^{j w t}+\right.$ c.c $) \mathscr{Q}_{0}^{\&}(t)$. Therefore, the average of dissipated power during one period $T=2 \pi /|\omega|$ of oscillation is calculated through the expression:

$$
P_{0}(w)=\frac{1}{2 p / w} \grave{\mathbf{O}}_{0}^{2 p / w} P_{0}(t) d t=\frac{1}{2 p / w} \grave{\mathbf{O}}_{0}^{2 p / w}\left(V_{0} e^{j w t}+\text { c.c }\right) \mathcal{L}_{0}^{\&}(t) d t
$$

Since we are interested to the response of the system near (or at the resonance), we assume $\left|\omega-\omega_{0}\right|=\omega$ and $\beta \omega \approx \beta \omega_{0}$. Under this approximation and using a few step of algebra, the electric power in Eq. (12) can be rewritten as:

$$
P_{0}(\omega) \approx j \frac{p_{0}}{\chi_{0}},
$$

where $j=\sqrt{-1}, p_{0}=\left|V_{0}\right|^{2} / r_{e 0}, \chi_{0}=\delta_{0} / \omega_{0}+j \beta_{0}, \delta_{0}=\omega_{0}-\omega$.

In Figs. 5(a) and 5(b), the real and imaginary parts of the steady state electric power $P_{0}(\omega)$ dissipated by the circuit are shown as a function of the normalized voltage frequency $\omega / \omega_{0}$, this for different values of the non-Hermitian gain/loss parameter $\beta_{0}$. The different plot parameters are shown in the figure where the solid lines represent the real parts of the normalized power $\left(\operatorname{Re}\left(P_{0} / p_{0}\right)\right)$ and the dashes/dots lines are corresponding to imaginary parts $\left(\operatorname{Im}\left(P_{0} / p_{0}\right)\right)$. In all plots, the green and blue curves correspond to the loss loop of which $\beta_{0}=0.5>0$ whereas purple and red colors refer to the gain loop with $\beta_{0}=-0.5<0$, respectively. As we can notice on the plots, when the natural frequency is negative $\left(\omega_{0}<0\right)$, the absorption profile presents a lorentzian form with the maximum of the absorption centered at $\omega=-\left|\omega_{0}\right|$ , while this lorentzian is rather centered at $\omega=\omega_{0}$ if the natural frequency is positive $\left(\omega_{0}>0\right)$. Remarkably, in the case of loss loop, these lorentzians are positive, corresponding to a typical absorption, whereas they are negative for the gain loop. Otherwise, in the latter case, the negative power was interpreted as the power amplified instead of being absorbed [41]. The imaginary part of the power corresponds to the dispersion and presents a positive slope around the absorption 
peaks in the case $\omega_{0}<0$ while the slope is negative when $\omega_{0}>0$. The results indicate that, to obtain the maximum of absorption (or amplification) at the resonance where it is expected, the voltage frequency should have the same sign and the same value as that of the natural loop frequency. Although negative frequencies have been suggested to many past researches [42-46], their using is often overlook in the context of analog model of EIT and related phenomena.

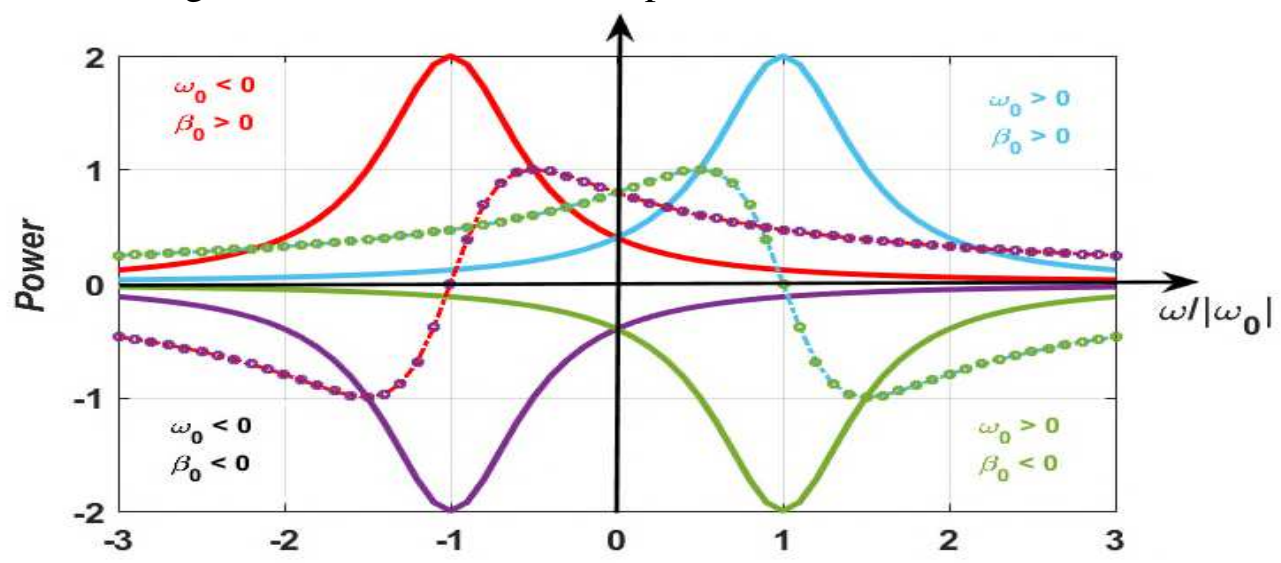

Fig. 5: The steady state normalized power dissipated by the $Z_{0} R_{0} C_{0}$ loop as function of the normalized frequency $\omega / \omega_{0}$ for different values of the non-Hermitian parameter $\beta_{0}$. The different plot parameters are shown in the figure. The solid lines represent the real part of the normalized power $\left(\operatorname{Re}\left(P_{0} / p_{0}\right)\right)$ and the dashes/dots lines correspond to the imaginary parts $\left(\operatorname{Im}\left(P_{0} / p_{0}\right)\right)$. In all plots, green and blue colors curves correspond to the loss loop ( $\beta_{0}=0.5$ ) whereas purple and red colors refer to the gain loop $\left(\beta_{0}=-0.5\right)$, respectively.

\subsection{Dynamic in a ZRC dimer}

From the eigenmodes of the ZRC dimer illustrated in Fig.2, we have identified two different coupling regimes: the weak and the strong coupling, border by the EPs, since the eigenvalues exhibit different behavior between and outside of the EPs. Another way to well distinguish the both regimes, is to investigate on the dynamic of the coupling oscillators. According to Eq. (3), the eigenvectors $A_{i+}, A_{i-}(i=0,1)$ describing the normal modes of the system satisfy to [47]:

$$
A_{1 \pm}=\alpha_{ \pm} A_{0 \pm},
$$

where $\alpha_{ \pm}=\frac{\omega_{0}+\left(j \beta_{0}-1\right) \omega_{ \pm}}{\Omega-\omega_{ \pm} \beta_{c}}=\frac{\Omega-\omega_{ \pm} \beta_{c}}{\omega_{0}+\left(j \beta_{1}-1\right) \omega_{ \pm}}, \beta_{C}=r_{C} / r, \Omega=1 / r C_{C}=\kappa \omega_{0}, r=r_{0,1}+r_{c}$ and $\omega_{0}=1 / r C$. The frequencies $\omega_{ \pm}$are found in Eq. (5).

If $c \neq c_{t h}=\beta_{0}(\varepsilon-1) / 2$, the general solution $Q_{0,1}(t)$ for the ZRC dimer can be written as:

$$
Q_{i}(t)=A_{i+} e^{j \omega_{+} t}+A_{i-} e^{j \omega_{-} t},
$$

where $i=(0,1)$. The amplitudes $A_{i \pm}$ are found from the initial conditions of the oscillators: $A_{0+}=\left(\alpha_{-} Q_{0}(0)-Q_{1}(0)\right) /\left(\alpha_{-}-\alpha_{+}\right)$and $A_{0-}=\left(Q_{1}(0)-\alpha_{+} Q_{1}(0)\right) /\left(\alpha_{-}-\alpha_{+}\right)$with $Q_{i}(0)$ being the initial charges of the capacitors. Then, we have calculated the 
amplitude squared $\left|Q_{i}\right|^{2}$ associated to each oscillator loop and the response is significantly describing the transfer of energy from one to another. Let us analyze the dynamic of both dimers $\mathrm{Z}$ and $\mathrm{ZC}$ (where $\kappa=0.75$ ). In the case of dimer ZC, the double coupling, offers the possibility to obtain a CPLP which is situated at the center of the weak region. Using for example the values $\varepsilon=0$ and $\beta_{0}=0.1$, the EPs are found to be $\beta_{C t h 1}=0.7$ and $\beta_{C i h 2}=0.8$ and the CPLP occurs at $\beta_{C}=0.75$. Hence the weak coupling is situated into the domain $\beta_{C} \in[0.7 ; 0.8]$ and the strong coupling regime is everywhere else. The use of dimer $\mathrm{Z}$ in the same conditions allows to situate the weak regime between $\beta_{C 1}=-0.05$ and $\beta_{C 2}=0.05$, while the strong regime is everywhere else. A rapid analysis of these range of values demonstrates for example that the multi coupling allows to reveal informations carried in the negative coupling values since the dimer $\mathrm{ZC}$ has shifted the weak domain of the dimer Z. In fig. 6, we have plotted the quantities $\left|Q_{0}\right|^{2}$ and $\left|Q_{1}\right|^{2}$, as a function of the time $t$ for different values of the gain/loss ratio $\varepsilon$, according to the driving regime. In addition, the initial conditions of the dimer are chosen such as $Q_{0}(0)=0, Q_{1}(0)=1$ , meaning that the signal is introduced into the coupling loop (oscillator 1 ).
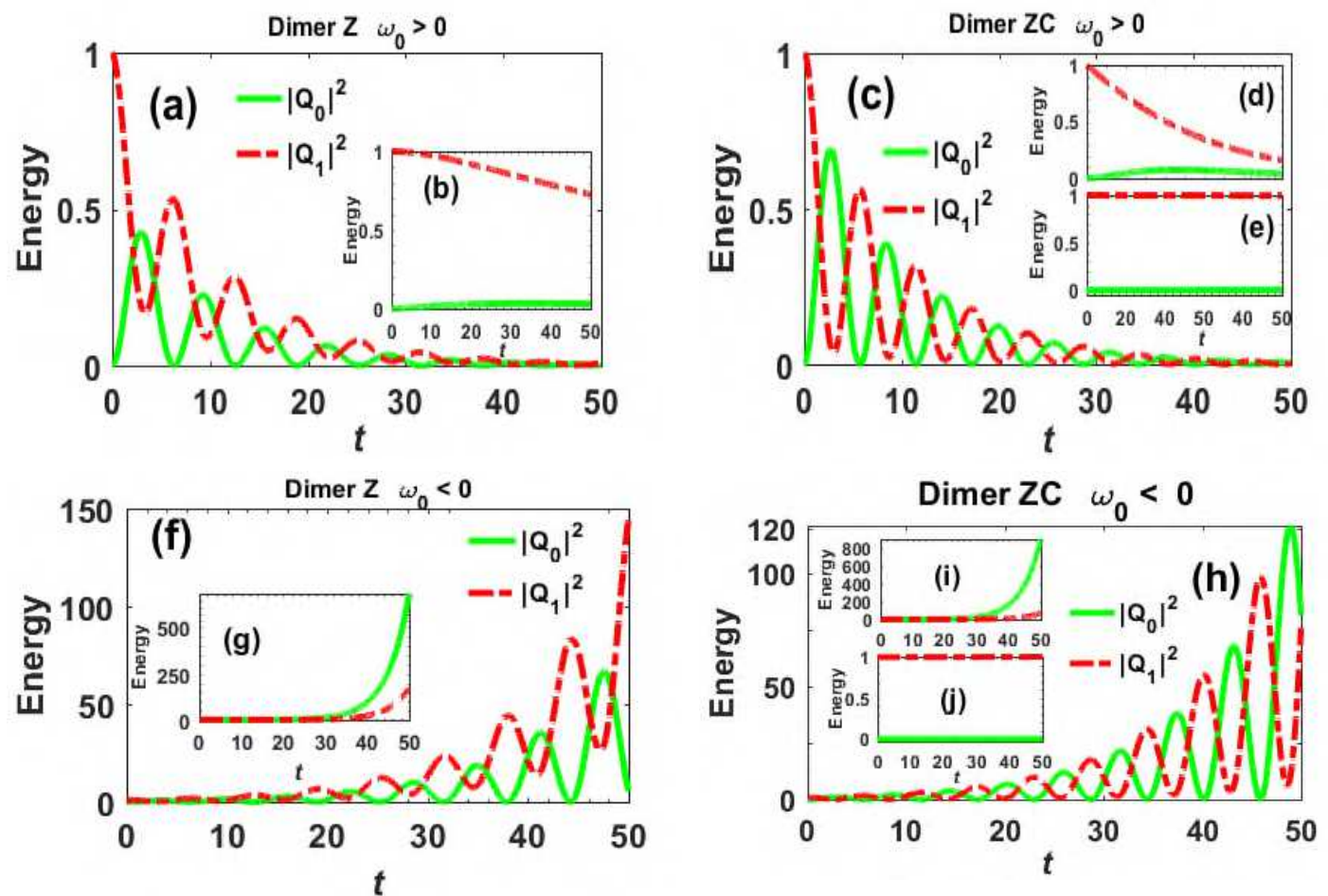

Fig. 6: Time evolution of the energy in each cell of the dimer Z (first column) and the dimer ZC when $\kappa=0.75$ (second column). (a) and (f): dimer $Z$ ( $\left.\beta_{C}=0.5\right)$ in strong regime. (b) and (g) correspond to dimer Z ( $\left.\beta_{C}=0.02\right)$ in the weak coupling regime. (c) and (h): dimer $\mathrm{ZC}\left(\kappa=0.75, \beta_{C}=0.2\right)$ in the strong regime. (d) and (i): dimer $\mathrm{ZC}\left(\kappa=0.75, \beta_{C}=0.71\right)$ corresponding to the weak coupling regime. (e) and (j) represent the dimer ZC at the $\operatorname{CPLP}\left(\kappa=\beta_{C}=0.75\right)$. From (a) to (e) $: \omega_{0}=1 \mathrm{rad} \cdot \mathrm{s}^{-1}$ and from (f) to (j) $: \omega_{0}=-1$ rad.s ${ }^{-1}$. For all plots $\varepsilon=0$ and $\beta_{0}=0.1$. The solid green and red dashes curves represent the energies $\left|Q_{0}\right|^{2}$ and $\left|Q_{1}\right|^{2}$, respectively. 
In the strong coupling regime, once a signal is put into oscillator 1, whatever for the dimer Z (Figs. 5(a) and 5(f)) or dimer ZC (Figs.5(c) and 5(h)), we note a rapid periodic exchange of energy between both oscillators loops which is made in an oscillatory process so that, when one is at its maximum, the other reaches its minimum. These oscillations are damped during the time in both oscillators when the frequency $\omega_{0}$ is positive while they are amplified if frequency is negative. Also notice that these oscillations are very fast with the dimer ZC. In the weak coupling regime, it can be remarked that when the frequency $\omega_{0}$ is positive, the energy is irreversibly transferred from oscillator 1 to oscillator 0 of which energy remains almost closed to zero whether in the case of the dimer Z (Fig. 5(b) or of the dimer $\mathrm{ZC} \mathrm{(Fig.} \mathrm{5(d)).} \mathrm{However,} \mathrm{if} \mathrm{the} \mathrm{frequency} \mathrm{is} \mathrm{negative,} \mathrm{once} \mathrm{the} \mathrm{signal} \mathrm{is} \mathrm{introduced}$ into oscillator 1 whether in the case of dimer Z (Fig. 5(g)) or dimer ZC (Fig. 5(i)), it takes a relatively long time to reach oscillator 0 , which then sees its energy exponentially increases while the amplitude of the master oscillator 1 grows slowly. At the CPLP belonging to the weak coupling regime, a specific behavior is observed. In fact, whether for $\omega_{0}$ positive (Fig. 5(e)) or negative (Fig. 5(j)), we remark that the signal introduced in oscillator 1 remains there and as consequence, no exchange of energy is made with the oscillator 0 whose amplitude remains zero.

In presence of the voltage, the exact solutions $Q_{i}(t)$ of Eq. (1) for the dimer can be written as a sum of two solutions: $Q_{0}(t)=Q_{0}^{c}(t)+Q_{0}^{p}(t)$, where the complementary solution $Q_{0}^{c}(t)$ linearly combines the two normal modes frequencies that is $Q_{0}^{C}(t)=A_{0_{+}} e^{j \omega_{+} t}+A_{0_{-}} e^{j \omega_{-} t}$ where $\omega_{ \pm}$are the eigenfrequencies obtained in Eq. (5) and $A_{o \pm}$ the complex amplitudes. The particular solution can be taken in the form of the voltage as $Q_{0}^{P}(t)=\rho_{0} e^{j \omega t}+$ c.c , with $\rho_{0}$ the amplitude. In the steady state regime in general, only the particular solution remains. Hence, replacing this solution into Eq. (12), the steady state power can be expressed as:

$$
P_{0}(\omega)=\frac{\left|V_{0}\right|^{2}}{r_{e 0}} \frac{j \omega\left(\delta+j \beta_{1} \omega\right)}{\left(\delta+j \beta_{0} \omega\right)\left(\delta+j \beta_{1} \omega\right)-\left(\Omega-\beta_{c} \omega\right)^{2}}
$$

where $\delta=\omega_{0}-\omega$ and $\Omega=\kappa \omega_{0}$.

Under the approximation $\left|\omega-\omega_{0}\right|=\omega, \beta \omega \approx \beta \omega_{0}$ and the expression of the steady state dissipated power can be rewritten as:

$$
P_{0}(\omega) \approx j \frac{p_{0} \chi_{1}}{\chi_{0} \chi_{1}-c^{2}}
$$

where $j=\sqrt{-1}, p_{0}=V_{0}{ }^{2} / r, c=\beta_{c}-\kappa, \chi_{0,1}=\delta / \omega_{0}+j \beta_{0,1}$ and $\delta=\omega_{0}-\omega$ is the frequency detuning between the loops frequencies' and the voltage frequency.

In the context of electronics, it is well known that the real part of the dissipated power (i.e. the active power) determines the absorption, while its imaginary part (i.e the reactive power) simulates the dispersion properties of the atomic medium. 
In Fig. 7, the normalized absorbed power $\left(P_{0} / p_{0}\right)$ of the main loop is reported as a function of the normalized voltage frequency $\omega / \omega_{0}$ according to the coupling strength regimes for dimer $\mathrm{ZC}$ and dimer $\mathrm{Z}$. The solid blue and the dashed red curves respectively represent the real $\left(\Re\left(P_{0}\right)\right)$ and the imaginary $\left(\Im\left(P_{0}\right)\right)$ parts of the power obtained when the main loop oscillator 0 is loss $\left(\beta_{0}=0.1>0\right)$ while the green curves presented in insets are obtained for the main loop gain $\left(\beta_{0}=-0.1<0\right)$. In addition, the natural frequency is assumed positive $\omega_{0}>0$ for the dimer ZC while for the dimer $\mathrm{Z}$, it is considered as negative $\omega_{0}<0$. By modulating the gain/loss ratio $\varepsilon$ from positive to negative values such as to maintain the system in a given coupling regime, we demonstrated that our system can replicate the features observed in EIT, EIA, ATS and related phenomena. For example, fixing the capacitive coupling $\kappa$ constant $(\kappa=0.75)$ for the dimer $\mathrm{ZC}$, we choose the values $\beta_{C}=0.71$ and $\beta_{C}=0.2$, leading the coupling in the weak and the strong regime, respectively. In absence of coupling (SW1 off), the absorption profile presents a lorentzian curve as discussed in Fig.5. Once a coupling is made between loops, it is remarked from Fig.7 (a) for a weak coupling $\left(\beta_{C}=0.71\right)$ that, a narrow dip appears in the spectrum when $\varepsilon=0.2$ leading to two absorption peaks. In the same time, the dispersion curve that we observed in Fig.5 is modified. If $\varepsilon=0$, a zero absorption, so a transparency window occurs in the spectrum. Then, the dispersion curve becomes much steeper near the zero central frequency. This behavior is similar to the EIT phenomenom interpreted for atomic systems, as a direct manifestation of the destructive interference between the normal modes of oscillations. The EIT phenomenon has been already replicated via LRC circuits [28]. On the contrary, when a small negative dissipation is introduced into the pumping oscillator 1 ( $\varepsilon=-0.1$ ), a negative absorption is observed in the transparency zone. Since a negative absorption refers to the amplification, we named this last behavior as electromagnetically induced amplified transparency (EIAT). For a further decrease of $\varepsilon$ until the value $\varepsilon=-0.18$, an enhanced absorption peak occurs in the profile where the dip was expected to be. Then, the system allows to mimic the absorption spectrum of three levels atom in lambda ( $\mathrm{L}$ ) configuration in the regime known as electromagnetically induced absorption (EIA). This EIA behavior has for it been interpreted for atomic systems as a manifestation of a constructive interference between the normal modes of oscillations.

Fig. 7 (b) shows the absorption profile of the dimer $\mathrm{ZC}$ considering the strong coupling $\left(\beta_{C}=0.2\right)$. It is observed for higher losses into the pumping field (for example when $\varepsilon=3$ ), two absorption peaks separated by a large transparency window. These peaks are clearly identified when the losses decrease to zero i.e when $\varepsilon=0$.The phenomenon is similar to the well-known Autler-Townes Splitting (ATS) effect which is generally interpreted as a consequence of a gap between two atomic resonances peaks. The peaks are located on both side of the central frequency $\omega_{0}$. A further decreasing of the damping that is, for a negative dissipation 
into the pumping oscillator 1 (i.e. $\varepsilon=-0.8$ ), a negative absorption occurs in the spectrum leading to the enhancement of the absorption peaks. On the contrary, when $\varepsilon$ less than -1, the peaks become inverted so that once a negative power (amplification) is observed in the profile. We termed this behavior as the AutlerTownes splitting with amplification (ATSA). All these results obtained with a positive frequency can be obtained with the negative voltage frequencies when $\omega_{0}<0$, as illustrated in fig. 7(d) in the case of dimer $\mathrm{Z}$ of which the set parameters are $\beta_{C}=0.02$ (weak coupling) and $\beta_{C}=0.5$ (strong coupling). This demonstrates the ability for such a circuit to offer a large range of investigations. Also notice that whether for the weak or the strong coupling, the plots in insets dictate what is observed if the main loop was gain.
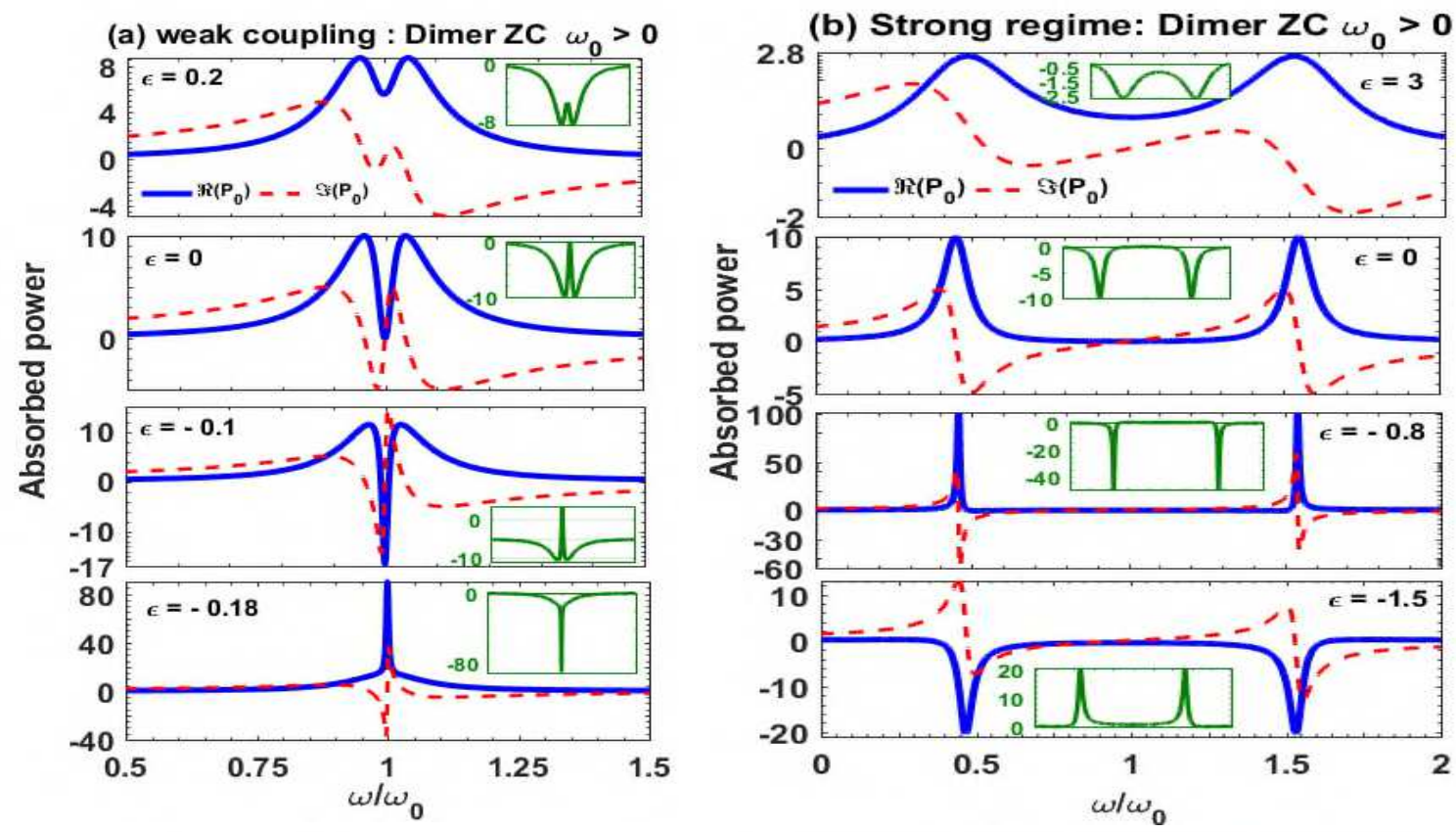

(c) Dimer ZC at CPLP $\forall \epsilon$
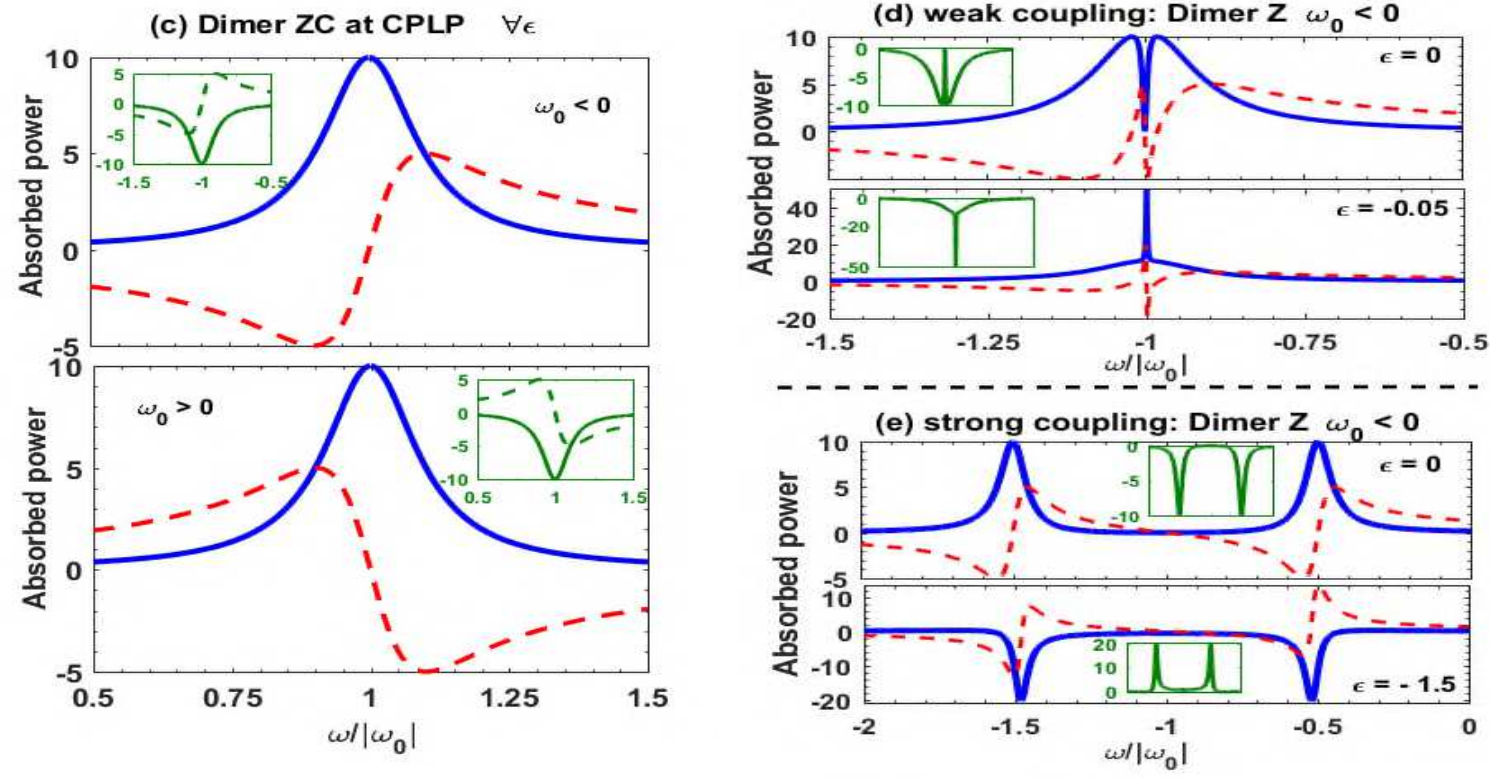

Fig.7: Absorbed power (dissipated) by the main loop of the dimer ZC (from (a) to (c)) when the natural frequencies of the loop are positive $\omega_{0}>0$ and the one of the dimer $Z$ (d) when frequencies are negative 
$\omega_{0}<0$. The solid blue and dashed red correspond to the real and imaginary parts of the absorbed power when the main loop is loss $\left(\beta_{0}=0.1>0\right)$. The insets in green show the real part of the power corresponding to the main loop gain $\left(\beta_{0}=-0.1<0\right)$. The other plot parameters are identical to those used in fig.5.

Fig.7 (c) illustrates the absorption at the CPLP of the dimer ZC ( $\left.\kappa=\beta_{C}=0.75\right)$, a particular point where the effective coupling cancels i.e $c=0$. Replacing the couplingless condition into Eq. (17), the expression of the absorbed power by the main loop of the dimer ZC is reduced to the expression of power given in Eq. (12) for the isolated cell. Hence, the cancellation of the effective coupling has effect to decouple the parts of the system and then, no exchange of energy can be observed. It results that no interference phenomenon can be obtained as it can be well confirmed by the plots which are simply the reproduction of what shown in Fig.3, where only lorentzian curves centered on the central frequency $\omega_{0}$ were observed. Accordingly, all the replicated atomic phenomena disappear. This is not a surprise for us, given the analysis made in the previous section about the transfer of energy in the CPLP. The results obtained here with the analogous circuit model, would be very interesting and could be an efficient way to control quantum information storage and the occurrence of the EIT windows in experiments with real atomic systems.

\subsection{Dynamic in a ZRC multi dimer}

Let us now focus on the ZRC multi dimer scheme when $N>1$, as depicted in Fig. 1(a). Such a system is used to replicate the multiple EIT, EIA or ATS windows as well for the positive as for the negative voltage source frequencies. It has been demonstrated [29] that, to obtain multiple transparency windows, each part of the circuit must oscillate with its own natural frequency $\omega_{i}(i=0 . . N)$, which differs from one loop to another. In presence of the voltage, Eq. (1) describing the ZRC multi dimer is reduced to Eq. (7) when rewriting it by incorporating the harmonic voltage $u(t)=V_{0} e^{j w t}+$ c.c in the right term. The amplitudes $A_{i}(i=0 \ldots N)$, can then be obtained and accordingly, the charge solution $Q_{0}(t)$ through the main loop. Thanks to Eq. (12), we calculated the general expression of the steady state frequency-dependence power $P_{0}(\omega)$, which is dissipated by the main loop as a function of the number $\mathrm{N}$ ( $\mathrm{N} \geq 1$ ) of the coupled loops in place. Under the approximation $|\omega-\varpi|=\omega, \beta_{i} \omega \approx \beta_{i} \varpi$ , where $\varpi=\omega_{0}$ is the mean frequency of the system, and using a few steps of algebra, the dissipated power $P_{0}(\omega)$ can be expressed in this case as:

$P_{0}(\omega)=\frac{j}{D(\omega)}\left(\prod_{l=1}^{N} \chi_{l}-(N-1) c^{N}-\sum_{n=2}^{N} \sum_{m=1}^{N-n+2} \sum_{h=m+1}^{N-n+3} \mathrm{~L} \sum_{v=m+u}^{N-n+u}(N-n) c^{N-n+1} \chi_{m} \chi_{h} \mathrm{~L} \chi_{v}\right)$,

where $(i=0 . . N), c_{i}=\beta_{c_{i}}-\kappa_{i}, \beta_{C_{i}}=r_{C} / r_{e i}, \kappa_{i}=\Omega_{i} / \omega_{0}, \chi_{i}=\delta_{i} / \omega_{0}+j \beta_{i}, \delta_{i}=\omega_{i}-\omega, \beta_{i}=R_{i} / r_{e i}$, $\omega_{i}=1 / r_{e i} C_{e i}, r_{e i}=r_{i}+r_{c}$ and $C_{e i}=C_{i} C_{C} /\left(C_{i}+C_{C}\right)$. The expression of the denominator $D(\omega)$ is found in Eq. (8) as: 


$$
\begin{aligned}
& D(\omega)=\prod_{i=0}^{N} \chi_{i}-N c^{N+1}-\sum_{p=1}^{N} \sum_{i=0}^{N-p+1} \sum_{k=i+1}^{N-p+2} \mathrm{~L} \sum_{s=i+q}^{N-p+q}(N-p) c^{N-p+1} \chi_{i} \chi_{k} \mathrm{~L} \chi_{s} \\
& 1 \leq p \leq N, 2 \leq n \leq N, \mathrm{~N} \geq 1, q \geq 2, u \geq 3
\end{aligned}
$$

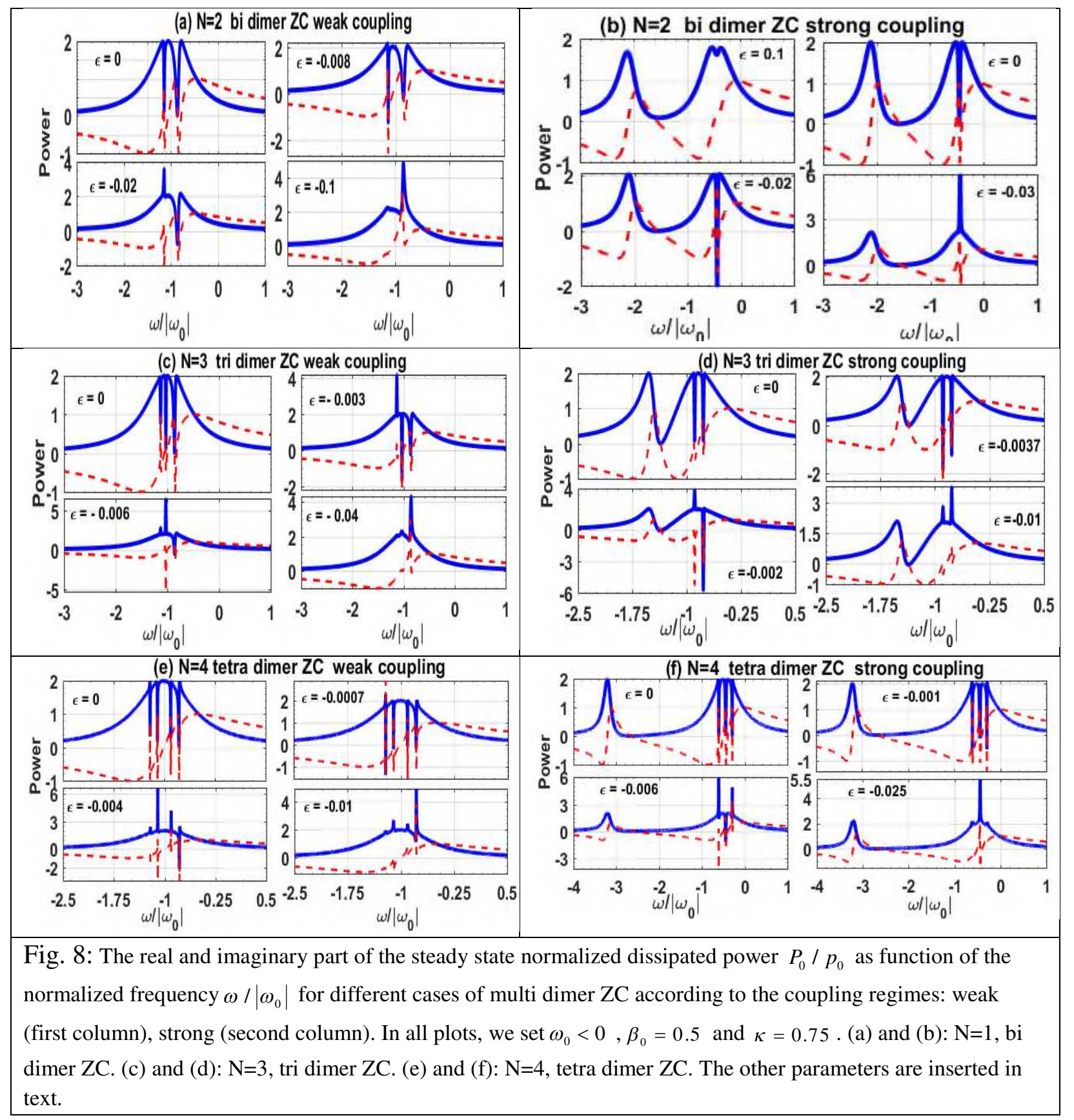

In Fig. 8, the real and the imaginary part of the dissipated power are reported as a function of the normalized frequency $\omega /\left|\omega_{0}\right|$ and the number $\mathrm{N}$ of the loops which are coupled to it, for different values of $\varepsilon$ parameter as indicated on the figure. As we can see, the graphs shows the dependence of its modulation with the absorption profile. For illustration, we have considered the natural frequencies of the loops same as those used in Fig. 4, i.e., for the bi dimer ZC ( $N=2): \omega_{1}=\omega_{0}-0.1 \omega_{0}$ and $\omega_{2}=\omega_{0}+0.1 \omega_{0}$; for the tri dimer ZC $(\mathrm{N}=3): \omega_{1}=\omega_{0}-0.1 \omega_{0}, \omega_{2}=\omega_{0}+0.02 \omega_{0}$ and 
$\omega_{3}=\omega_{0}+0.1 \omega_{0}$ while for the tetra dimer ZC $(\mathrm{N}=4): \omega_{1}=\omega_{0}-0.1 \omega_{0}, \omega_{2}=\omega_{0}+0.1 \omega_{0}$ $\omega_{3}=\omega_{0}-0.2 \omega_{0}$ and $\omega_{4}=\omega_{0}+0.2 \omega_{0}$, where $\omega_{0}$, the mean frequency is the natural frequency of the main loop which is taken negative here $\left(\omega_{0}<0\right)$. In addition, the main loop is considered as a loss loop $\left(\beta_{0}=0.5>0\right)$. In all plots we set $\kappa=0.75$. For the imaginary resistive coupling parameter $\beta_{C}$ taken near this value, the coupling is in the weak regime (left column), whereas for $\beta_{C}$ taken far away this value, the coupling is in the strong regime (right column).

Specifically, for the bi dimer ZC, the coupling parameters are $\beta_{C}=0.85$ (weak regime) and $\beta_{c}=0.2$ (strong regime). For a weak coupling, see Fig. 8(a), we notice in the absorption spectrum when $\varepsilon=0$, two symmetric dips centered at $\omega=0.9 \omega_{0}$ and $\omega=1.1 \omega_{0}$ on both sides of the central peak, thus leading two EIT windows. This behavior is similar to the quantum well known phenomenon of double electromagnetically induced transparency (DEIT). This behavior was also replicated in ref. [29] using LRC circuits. Accordingly, two steep slopes are observed from the dispersion curve in the transparency domain. Decreasing $\varepsilon$ to the value $\varepsilon=-0.008$, a phenomenon similar to the EIAT appears in the absorption spectrum however in two points where the negative power is automatically observed. Accordingly, we called this new behavior as double EIAT (DEIAT). For a further decreasing of $e$, to -0.002 and then to the value -0.1 , it is remarked in the spectrum the occurrence of one EIA peak where the first EIT dip was observed and then another one, where the second dip was expected to be. As consequence it results two enhanced absorbed peaks. Then, the bi dimer circuit allows to replicate double EIA peaks. We then termed this as double EIA (DEIA).

In the strong coupling regime (see Fig. 8 (b)), when a dissipation is introduced into the coupling field $(\varepsilon=0.1)$, it is observed two dips inside the profile, one of which is more pronounced and centered to the frequency $\omega_{0}$ and the other which is narrow. Both dips are separated by a large transparency domain, similar to the ATS effect described above. The location of the peaks is in perfect accordance with the discussions made for the eigenspectrum (see Fig. 4) where two frequencies are closed together while the other is far away. A further decrease of $\varepsilon$ to the value $\varepsilon=0$ reduces the dip to a zero absorption, leading to the phenomenology of EIT. So that the system allows to reproduce an EIT and an ATS window instead of two ATS windows as we could imagine. However, as one would expect, by lowering $\varepsilon$ to the negative values for example $\varepsilon=-0.02$, a negative power typical to the EIAT effect described above in the case $\varepsilon=0$ occurs in the transparent region between the two EIT peaks. For $\varepsilon=-0.03$, it is not a surprise to note that the spectrum just pass from the EIAT to the EIA behavior as in the case of the dimer ZC. In all cases, it is noted that the dispersion profile is modified according to the type of phenomenon reproduced.

In the case of tri dimer $\mathrm{ZC}(\mathrm{N}=3)$, it is remarked from Fig. 8(c) for the coupling in the weak domain ( $\beta_{C}=0.803$ ), that the system allows to reproduce when 
$\varepsilon=0$, three narrow dips centered at the naturel loops frequencies $0.9 \omega_{0}, 1.02 \omega_{0}$ and $1.1 \omega_{0}$, thus exhibiting three EIT windows, a behavior similar to the Triple EIT (TEIT) which was observed in atomic context [48, 49] and studied in a superconducting quantum circuit with a four-level V-type energy spectrum [55]. Similarly, a further decreasing of $\varepsilon$ to -0.003 , then to -0.006 and finally to -0.04 allows to the spectrum to switch from Triple EIAT (TEIAT) to double EIA (DEIA) and then to Triple EIA (TEIA), respectively. However, for a strong coupling ( $\left.\beta_{C}=0.58\right)$, the observations made in Fig. 8(d) when the gain/loss ratio takes respectively the values $\varepsilon=0, \varepsilon=-0.0037, \varepsilon=-0.002$, and $\varepsilon=-0.01$, are similar to the ones obtained with the bi dimer $\mathrm{ZC}(\mathrm{N}=2)$, however with a consequent increasing of the number of EIT, EIAT dips or EIA peaks but with an invariable number of ATS window, i.e. one.

In the case of the tetra dimer $\mathrm{ZC}(\mathrm{N}=4)$, in presence of the weak coupling ( $\left.\beta_{C}=0.78\right)$, it is observed when $\varepsilon=0$, four EIT windows, that we named as quadruple EIT (QEIT). By playing on the value of $\varepsilon$, our model allowed us to also reproduce a quadruple EIAT (QEIAT) windows when $\varepsilon=-0.0007$, then a TEIA when $\varepsilon=-0.004$ and finally a quadruple EIA (QEIA) if $\varepsilon=-0.01$. In presence of the strong coupling (with $\beta_{C}=0.2$ ), the tetra dimer $\mathrm{ZC}(\mathrm{N}=4)$ allows also to mimic TEIT $(\varepsilon=0)$, TEIAT $(\varepsilon=-0.001)$, DEIA $(\varepsilon=-0.006)$ and TEIA $(\varepsilon=-0.0025)$ accompanied in each case by the ATS window alone.

To generalize, the ZRC multi dimer is able to replicate N-EIT, N-EIAT or N-EIA windows in the weak coupling regime, the number of windows depending on the number $\mathrm{N}$ of loops coupled to the main loop of which the voltage source acts directly. In the same way, in the strong coupling, the interplay between the control and the probe field induce a simultaneous one ATS, (N-1) EIT, (N-1) EIAT or (N1) EIA windows. It is also important to recall that, these results are obtained here with a negative main loop frequency and a positive value of $\beta_{0}$. They can also be reproduced as shown in Fig.5, at positive frequencies and even also when the parameter of non-hermiticity $\beta_{0}$ is negative. In this last hypothesis, all phenomena reproduced here should be amplified. Finally it is important for us to notice that at the couplingless point (CPLP) of any multi dimer ZC, all the mimicked phenomena disappear as discussed in Fig.7(c).

\section{Conclusion}

In summary, we have proposed a non-Hermitian electronic dimers based on the imaginary resistor, that mimics a $(\mathrm{N}+2)$ level atomic system in multi-pod type configuration. The equations describing the so called ZRC multi dimer circuit were theoretically analyzed under the resonance conditions considering as well positive as negative frequencies. The eigenanalysis allowed us to identify two coupling strengths; the weak and the strong coupling regimes. Thereafter, we have demonstrated that in the weak coupling regime, our system is able to replicate multiple EIT, EIAT and EIA. In the presence of the strong coupling, the system 
also allows to mimic the quantum phenomena mentioned above in addition to the ATS-like effect. Remarkably, we have illustrated a particular point of the weak coupling regime where the system becomes couplingless as its effective coupling cancels. The analysis of the frequencies splitting reveals that at the couplingless point (CPLP), the systems seems as decoupled and consequently there is no exchange of energy between parts of the circuit. It comes out that all the replicated phenomena disappears. Finally, it is important to mention that to reproduce EIT, ATS or EIA and related phenomena with electronic circuit does not means that these phenomena are classical. In addition to show the analogy between the electronic circuit and the atomic system, our work well provides a description and observation of quantum interference processes happening in atomic media. This intuitively suggests to integrate a concept of non-hermiticity, a multiple coupling and the negative frequencies in modeling quantum phenomena. Thanks to the use of the imaginary resistance, the equations describing the ZRC multi dimer were easily manipulated. We hope that it will be a better prototype that can help students and researchers in understanding quantum multiple phenomena observable in atomic media and could find further ideas for futures works.

\section{Acknowledgements}

Authors would like here to acknowledge TABEU Stéphane Boris of HITASTEC (High-tech and Slow Technology) for helpful discussions.

\section{References}

1. S. E. Harris, J. E. Field, and A. Imamoğlu, "Nonlinear optical processes using electromagnetically induced transparency,” Phys. Rev. Lett. 64, 1107-1110 (1990)

2. S. E. Harris, “Electromagnetically induced transparency,” Phys. Today, 50, 36-42 (1997)

3. M. O. Scully, "From lasers and masers to phaseonium and phasers," Phys. Rep., 219, 191 (1992)

4. A. B. Matsko, Y. V. Rostovtsev, M. Fleischhauer, and M. O. Scully, "Anomalous Stimulated Brillouin Scattering via Ultraslow Light,” Phys. Rev. Lett., 86, 10, 2006-2009 (2001).

5. M. Fleischhauer, A. Imamoglu, and J. P. Marangos, “Electromagnetically induced transparency: Optics in coherent media,’' Rev. Mod. Phys. 77, 633-673 (2005).

6. S. H. Autler and C. H. Townes, “Stark effect in rapidly varying fields,' Phys. Rev. 100, 703$722(1955)$

7. C. N. Cohen-Tannoudji, "The Autler-Townes Effect Revisited," Amazing Light, 109-123 (1996)

8. J. Mompart and R. Corbalán, “Lasing without inversion,” J. Opt. B, 2 , R7 - R24 (2000)

9. K. Totsuka, N. Kobayashi, and M. Tomita, "Slow light in coupled-resonator-induced transparency," Phys. Rev. Lett. 98, 213904 (2007).

10. C. Liu, Z. Dutton, C. H. Behroozi, and L. V. Hau, "Observation of coherent optical information storage in an atomic medium using halted light pulses,” Nature, 409, 490-493 (2001).

11. D. F. Phillips, A. Fleischhauer, A. Mair, and R. L. Walsworth, "Storage of light in atomic vapor," Phys. Rev. Lett. 86, 783-786 (2001). 
12. G. Hétet, A. Peng, M. T. Johnsson, J. J. Hope, and P. K. Lam, "Characterization of electromagnetically-induced-transparency-based continuous-variable quantum memories," Phys. Rev. A 77, 012323 (2008).

13. H. Wang, D. Goorskey, and M. Xiao, "Enhanced Kerr Nonlinearity via Atomic Coherence in a ThreeLevel Atomic System,” Phys. Rev. Lett. 87, 0736601 (2001)

14. N. Liu, T. Weiss, M. Mesch, L. Langguth, U. Eigenthaler, M. Hirscher, C. Sönnichsen, and H. Giessen, "Planar Metamaterial Analogue of Electromagnetically Induced Transparency for Plasmonic Sensing," Nano Lett. 10, 1103-1107 (2010)

15. P. Bermel, A. Rodriguez, S. G. Johnson, J. D. Joannopoulos, and M. Soljačić, "Single-photon alloptical switching using waveguide-cavity quantum electrodynamics," Phys. Rev. A 74, 043818 (2006).

16. A. Lezama, S. Barreiro, and A. M. Akulshin, "Electromagnetically induced absorption," Phys. Rev. A 59, 4732-4735 (1999)

17. R. Ramzan, O. F. Siddiqui, M. W. Arshad, and O. M. Ramahi, “A Complex permittivity extraction method based on anomalous dispersion,” IEEE Trans. on Microw. Theory Tech. 64, 3787-3796 (2016).

18. O. Siddiqui, R. Ramzan, M. Amin, and O. M. Ramahi, “A non-invasive phase sensor for permittivity and moisture estimation based on anomalous dispersion,” srep28626 (2016).

19. R. Ramzan, M. Omar, O. F. Siddiqui, M. Amin, N. Bastaki, and T. S. Ksiksi, "Electromagnetically induced absorption in the near-field of microwave radiative elements with application to foliage moisture sensing," IEEE Access, 6,77859-77868 (2018)

20. Y. Wang, C. Xue, Z. Zhang, H. Zheng, W. Zhang, and S. Yan, "Tunable optical analog to electromagnetically induced transparency in graphene-ring resonators system,” Sci. Rep., 6, 1 (2016).

21. D. D. Smith, H. Chang, K. A. Fuller, A. T. Rosenberger, and R. W. Boyd, “Coupled-resonator-induced transparency," Phys. Rev. A, 69, 6 (2004).

22. K. R. Dastidar and S. Dutta, "Broadening of EIT window by incoherent pumping in a three-level $\Lambda$ system: Effect of homogeneous and inhomogeneous broadening,” EPL., 82, 54003 (2008).

23. D. Petrosyan and Y. P. Malakyan, "Magneto-optical rotation and cross-phase modulation via coherently driven four-level atoms in a tripod configuration,” Phys. Rev. A, 70, 023822 (2004)

24. B. N. Huy, D. Le Van, and K. D. Xuan, "Controllable optical properties of multiple electromagnetically induced transparency in gaseous atomic media," Commun. Phys., 29, 1 (2019).

25. V. Bharti and A. Wasan, "Complete wavelength mismatching effect in a Doppler broadened Y-type six-level EIT atomic medium,” Opt. Commun., 324, 238-244 (2014).

26. E. Paspalakis and P. L. Knight, "Electromagnetically induced transparency and controlled group velocity in a multilevel system", Phys. Rev. A 66, 015802 (2002)

27. D. McGloin, D. J. Fulton, and M. H. Dunn, "Electromagnetically induced transparency in N-level cascade schemes," Opt. Commun., 190, 221-229 ( 2001)

28. C. L. Garrido Alzar, M. A. G. Martinez, and P. Nussenzveig, "Classical analog of electromagnetically induced transparency,” Am. J. Phys. 70, 37-41 (2002).

29. J. Harden, A. Joshi, and J. D. Serna, "Demonstration of double EIT using coupled harmonic oscillators and RLC circuits,” Eur. J. Phys. 32, 541-558 (2011). 
30. Z. Bai, C. Hang, and G. Huang, "Classical analogs of double electromagnetically induced transparency," Opt. Commun. 291, 253-258, (2013).

31. S. Tagouegni, F. Fotsa-Ngaffo, S. B. Tabeu, and A. Kenfack-Jiotsa, "Energy transport and Andersonlike localization in non-Hermitian electrical transmission line,” Phys. Scr., 95, 9, 095808 (2020).

32. S. B. Tabeu, F. Fotsa-Ngaffo, and A. Kenfack-Jiotsa, "Imaginary resistor based Parity-Time symmetry electronics dimers," Opt. Quant. Electron. 51, (2019).

33. M. Frimmer and L. Novotny, "The classical Bloch equations," Am. J. Phys., 82, 947-954 (2014).

34. K. Shouno and Y. Ishibashi, "Synthesis and active realization of a three-phase complex coefficient filter using gyrators,” 2008 51st Midwest Symposium on Circuits and Systems , (2008)

35. T. Fujii and K. Shouno, "Realization of Impedance-scaled Complex Filters Using Reciprocal Elements," 2019 34th International Technical Conference on Circuits/Systems, Computers and Communications (ITC-CSCC), (2019).

36. T. Nakagawa, K. Shouno, and K. Kuniya, "Synthesis of a first-order passive complex filter with bandpass/band-elimination characteristics,” Analog Integr. Circuits Signal Process., 78, 33-42 (2013).

37. A. S. Elwakil and B. J. Maundy, "Indirect Realization of the Imaginary Resistor jR," Analog Integr. Circuits Signal Process, 35, 2610-2615 (2015).

38. J. Schindler, Z. Lin, J. M. Lee, H. Ramezani, F. M. Ellis, and T. Kottos, "PT-symmetric electronics", J. Phys. A: Math. Theor. 45, 444029 (2012)

39. F. Fotsa-Ngaffo, S. B. Tabeu, S. Tagouegni, and A. Kenfack-Jiotsa, "Thresholdless characterization in space and time reflection symmetry electronic dimers,” J. Opt. Soc. Am. B, 34, 658 (2017).

40. S. R.-K. Rodriguez, “Classical and quantum distinctions between weak and strong coupling,” Eur. J. Phys., 37, 025802 (2016)

41. L. Hai-Chao and G. Guo-Qin, "Switching from positive to negative absorption with electromagnetically induced transparency in circuit quantum electrodynamics”, Chin. Phys. 23, 054206 (2014)

42. E. Rubino, J. McLenaghan, S. C. Kehr, F. Belgiorno, D. Townsend, S. Rohr, C. E. Kuklewicz, U. Leonhardt, F. König, and D. Faccio, “Negative-Frequency Resonant Radiation,” Phys. Rev. Lett. 108, 253901 (2012)

43. M. Conforti, N. Westerberg, F. Baronio, S. Trillo, and D. Faccio, "Negative-frequency dispersive wave generation in quadratic media," Phys. Rev. A, 88, 013829 (2013).

44. C. R. Lourés, D. Faccio, and F. Biancalana, "Nonlinear Cavity and Frequency Comb Radiations Induced by Negative Frequency Field Effects,” Phys. Rev. Lett., 115, 193904 (2015).

45. S. Pendharker, Y. Guo, F. Khosravi, and Z. Jacob, "PT-symmetric spectral singularity and negativefrequency resonance,” Phys. Rev. A 95, 033817 (2017).

46. M. V. Nezlin, "Negative-energy waves and the anomalous Doppler effect,” Sov. Phys. Uspekhi, 19, 946-954 (1976)

47. G. Dolfo and J. Vigué, "Damping of coupled harmonic oscillators,” Eur. J. Phys, 39, 025005 (2018)

48. T. Wang, Y.-Q. Hu, C.-G. Du, and G.-L. Long, "Multiple EIT and EIA in optical microresonators," Opt. Expr., 27, 7344 (2019).

49. G. Tiaz, E. Shahaliyev, M. A. Ashiq, and F. Ghafoor, "Multiple Color Electromagnetically Induced Switching Using a Five-Level Atomic Medium,”J. Quant. Electron. 55, 1-10 (2019). 